\title{
Ultimate Limits for Counterweight Balancing of Crank-Rocker Four-Bar Linkages
}

\author{
Bram Demeulenaere*, Erwin Aertbeliën, Myriam Verschuure, \\ Jan Swevers, Joris De Schutter \\ Katholieke Universiteit Leuven, Department of Mechanical Engineering \\ Celestijnenlaan 300B, B-3001 Heverlee, Belgium
}

\begin{abstract}
This paper focuses on reducing the dynamic reactions (shaking force, shaking moment and driving torque) of planar crank-rocker four-bars through counterweight addition. Determining the counterweight mass parameters constitutes a nonlinear optimization problem, which suffers from local optima. This paper, however, proves that it can be reformulated as a convex program, that is, a nonlinear optimization problem of which any local optimum is also globally optimal. Because of this unique property, it is possible to investigate (and by virtue of the guaranteed global optimum, in fact prove) the ultimate limits of counterweight balancing. In a first example a design procedure is presented that is based on graphically representing the ultimate limits in design charts. A second example illustrates the versatility and power of the convex optimization framework by reformulating an earlier counterweight balancing method as a convex program and providing improved numerical results for it.
\end{abstract}

${ }^{*}$ Corresponding author. E-mail: bram.demeulenaere@mech.kuleuven. be 


\section{Introduction}

Dynamic balancing is the art of reducing (or eliminating) the dynamic reactions resulting from the rotary-to-oscillating motion conversion imposed by a mechanism. The dynamic reactions, considered here and formally defined in Sec. 3.1, are (i) the shaking force and (ii) shaking moment transmitted to the supporting frame, as well as (iii) the input or driving torque, required to drive the mechanism with constant drive speed. The motivation for dynamic balancing is twofold. First, shaking moments and shaking forces cause vibrations of the supporting frame (which in turn cause noise, wear, fatigue, ...), and should therefore be minimized. Second, highly peaked driving torques necessitate large flywheels (compromising the machine's start/stop behavior) or high actuator torques (and hence a large motor) in order to obtain a constant drive speed.

Spring addition and counterweight addition constitute two prominent dynamic balancing approaches. This paper focuses on counterweight addition for dynamically balancing (or for short, counterweight balancing, $C W B$ ) planar crank-rocker four-bar mechanisms. An elaborate introductory literature survey of CWB is not given here. The newcomer in the field is referred to the essential paper [1], and the comprehensive surveys [2,3].

It is well-known, see for instance $[4,5,6]$, that CWB involves a trade-off between minimizing the different dynamic reactions. A typical engineering way of handling these trade-offs is to incorporate them into an optimization problem. Before the advent of vast computational resources, analytic or semi-analytic optimization approaches were developed, exclusively devoted to fourbar mechanisms $[7,8,9,10,11,12]$. Gradually the focus has shifted to numerical optimization techniques $[13,14,15,16,17,18,19,20,21,22,23]$, which can cope with more complicated mechanisms, and allow a more flexible problem formulation. However, the resulting numerical optimization problems are generally considered to be difficult to solve, because of their nonlinear nature, which results in the presence of (an unknown number of) local optima. As a result, the designer is likely to end up, often after a great deal of computation, with a design that is typically not globally optimal. Conversely, the present paper proves that counterweight optimization can be reformulated as a convex program, that is, a (special kind of) nonlinear optimization problem of which any local optimum is also globally optimal. As a result, convex programs can be solved (implying that the global optimum is found) numerically with great efficiency [24].

The guarantee to obtain the global optimum allows us to prove, albeit in a numerical way, the ultimate limits of CWB. Ultimate implies here that the constraints imposed on the counterweight parameters are as little restrictive as possible. First of all, the mass $m_{i}^{*}[\mathrm{~kg}]$, centroidal moment 
of inertia $J_{i}^{*}\left[\mathrm{~kg}-\mathrm{m}^{2}\right]$ and center of gravity coordinates $\left(X_{i}^{*}, Y_{i}^{*}\right)[\mathrm{m}]$ of the $i$-th counterweight parameters $(i=1 \ldots 3)$ are used directly as the optimization variables. This implies that, as opposed to many balancing papers, no particular counterweight shape is assumed beforehand.

Second, the only constraints imposed on these optimization variables all have a physical nature: mass and centroidal moment of inertia must be nonnegative. Such constraints result in point-mass counterweights for links that have nonzero angular acceleration, like in $[12,14,16]$. Based on the notion of minimum inertia counterweights [25, 26], a simple procedure is proposed to convert the ultimate, point-mass counterweights into practical counterweights at the cost, of course, of slightly worse balancing results.

To the best of our knowledge, the only balancing optimization method that results in a guaranteed global optimum, like in the framework presented here, is the analytical method of Tepper and Lowen [9]. Tepper and Lowen's method is based on analytically manipulating the so-called KarushKuhn-Tucker optimality conditions [27], in order to obtain a polynomial equation of which the roots provide all local optima for their particular formulation of four-bar $\mathrm{CWB}^{1}$. The global optimum is then selected as the best of all local optima. The main obstruction for applying this method to other variants ${ }^{2}$ of the four-bar CWB problem is the symbolic manipulation required, which involves 'a great deal of computation' [9], as opposed to the method presented here, which is inherently numerical in nature, very flexible, and easily applicable to more complicated, planar mechanisms, as shown in [28].

The paper is organized as follows. First an introduction to convex optimization is given (Sec. 2). Section 3 formulates (one variant of) the ultimate CWB design problem, which turns out to be nonconvex in the optimization variables $m_{i}^{*}, J_{i}^{*}, X_{i}^{*}$ and $Y_{i}^{*}, i=1 \ldots 3$. After that, Sec. 4 introduces a nonlinear change of variables that allows reformulating this nonconvex problem as an equivalent, convex optimization problem. Based on a numerical example, Sec. 5 introduces a design procedure that is based on graphically representing the ultimate limits in design charts. Section 6 illustrates the versatility and power of the proposed framework, by (i) reformulating Sadler and Mayne's [14] variant of ultimate CWB as a convex problem, and (ii) presenting numerical results that improve those presented in [14]. Section 7 finally discusses the obtained results.

\footnotetext{
${ }^{1}$ Tepper and Lowen optimize minimum inertia counterweights attached to the crank and rocker of a crankrocker four-bar, so as to minimize the root-mean-square of the shaking force, subject to an upper limit on the root-mean-square of the ground bearing forces.

${ }^{2}$ For instance, if other constraints, like upper limits on the root-mean-square driving torque, are considered.
} 


\section{A Convex Optimization Primer}

In fact, the great watershed in optimization isn't between linearity and nonlinearity, but between convexity and nonconvexity [29]. The first part of this citation expresses a common misconception among engineers: if either the objective function or the constraints of an optimization problem are nonlinear, the problem is difficult to solve. Indeed, linear programs, that is, optimization problems with an objective function and constraints that are linear in the optimization variables, have nice properties: every local optimum is also globally optimal and there exist effective algorithms (like the well-known simplex method or the more recently developed interior point methods) that can reliably solve (that is, determine the global optimum of) even large problems, with hundreds or thousands of variables and constraints. Therefore, many engineers consider 'linear' and 'easy' on the one hand, and 'nonlinear' and 'difficult' on the other hand, as being synonyms in the area of optimization.

This is not quite true, as suggested by the second part of Rockafellar's citation: although convex programs ( $\mathrm{CPs}$ ) are nonlinear optimization problems, they do have the property that any local optimum is also globally optimal. Furthermore, very efficient and reliable algorithms exist (such as interior point methods) for solving CPs. As with linear programming, it can be said, with only a bit of exaggeration, that, if a problem can be formulated as a convex program, the original problem has been solved [24]. Formulating an optimization problem as a CP, therefore, has great advantages.

Unfortunately, recognizing convex optimization problems, or those that can be transformed into $\mathrm{CPs}$, is not straightforward: the art and challenge in convex optimization is in problem formulation. Once a problem is formulated as a convex program, it is relatively straightforward to solve it. In dealing with nonlinear programs (that is, nonlinear optimization problems that are not, or can't be proven to be, convex), things are reversed: formulating the optimization problem is mostly relatively straightforward. Here, however, the art and challenge is in solving the optimization problem. This involves testing different optimization algorithms, tuning the parameters of the chosen optimization algorithm (this can itself be an optimization problem), developing a methodology to produce a good enough initial guess,...

In the area of CWB, most work that focuses on numerical optimization adopts the latter, 'solvingoriented' approach $[13,14,15,16,17,19,20,21,22,23]$, often using so-called linearly independent vector expressions (introduced in [1] and discussed in Sec. 4.2 and Appendix A) for efficient numerical evaluation of the objective function and constraints. A notable exception constitutes 
the work of Tricamo and Lowen [18], in which a nonlinear program is obtained, but the efficiency of solving it (in the sense of obtaining a local optimum: the program is not convex) is greatly enhanced by using a technique, well-known in convex programming. That is, to replace the nonlinear program

$$
\operatorname{minimize}_{x} \max \left(f_{1}(x), f_{2}(x), \ldots, f_{m}(x)\right),
$$

where $x \in \mathbb{R}^{n}$ is the optimization variable vector and $f_{1}, \ldots, f_{m}$ are functions $\mathbb{R}^{n} \rightarrow \mathbb{R}$, by the equivalent nonlinear program

$$
\begin{aligned}
\operatorname{minimize}_{[x ; q]} & q \\
\text { subject to } & f_{i}(x) \leq q, i=1, \ldots, m
\end{aligned}
$$

The nonlinear program (1) has a nondifferentiable objective function, due to the presence of the max-function. The nonlinear program (2a)- (2b), in which an auxiliary variable $q$ is introduced, has a differentiable (even linear) objective function (2a), and nonlinear functions $f_{i}, i=1 \ldots m$ describing its constraints ( $2 b)$. If the latter functions are differentiable, the nonlinear program (2a)(2b) can be solved using a much broader and much more efficient class of optimization algorithms than the original program (1).

Presently, the more prominent application areas of convex optimization include [24] automatic control systems, estimation and signal processing, communication and networks, electronic circuit design, data analysis and modeling, statistics, and finance, whereas the number of applications in mechanical engineering is rather limited. Convex programming is well-known in truss-structure optimization [30,31], while many software packages for structural optimization are based on convex approximation methods [32]. Other mechanical applications of convex programming include grasping force optimization [33] and design of cantilever beams [34].

Several classes of CPs exist, each of which are a subclass of a more general type of problems. Starting with the more restrictive class, we have: linear programs (LPs), convex quadratic programs (QPs), second-order cone programs (SOCPs) and semidefinite programs (SDPs):

$$
\mathrm{LP} \subset \mathrm{QP} \subset \mathrm{SOCP} \subset \mathrm{SDP} \subset \mathrm{CP} .
$$

SOCPs are of particular interest here. In an SOCP, one minimizes a linear objective function, subject to linear equality constraints, linear inequality constraints and second-order cone constraints [24]. The latter constraints are of the general form:

$$
\|A \cdot x+b\|_{2} \leq c^{T} x+d,
$$


where $x \in \mathbb{R}^{n}$ is the optimization variable vector, and $A \in \mathbb{R}^{k \times n}, b \in \mathbb{R}^{k}, c \in \mathbb{R}^{n}$ and $d \in \mathbb{R}$ constitute given problem data. $\|\cdot\|_{2}$ denotes the $L 2$-norm: $\|x\|_{2}=\sqrt{x^{T} \cdot x}$. From here on, vectors and matrices are denoted using bold characters to distinguish them from scalars.

\section{Original Optimization Problem}

\subsection{Definition of Dynamic Reactions}

Figure 1(a) shows the kinematic scheme of a planar crank-rocker four-bar. All links are assumed to be rigid. Link 1 , the crank $p q$, is assumed to rotate at constant speed $\omega[\mathrm{rad} / \mathrm{s}]$, which determines the mechanism's period of motion $T=2 \pi / \omega[\mathrm{s}]$. Link 2 , the coupler $q r$, connects the crank with link 3 , the rocker $s r$. The latter performs an oscillating (rocking) motion. Link 4 is the fixed ground $p s . \phi_{i}(t)[\mathrm{rad}], i=\{1,2,3,4\}$ measure the link angle counterclockwise from the horizontal. $\phi_{4}[\mathrm{rad}]$ is a constant angle, which is of no importance if gravity is neglected. The latter, classical assumption in the area of high-speed linkages is also made here. The link lengths are denoted as $a_{i}[\mathrm{~m}], i=\{1,2,3,4\}$.

Figure $1(b)$ shows the corresponding free-body diagram, based on which the dynamic reactions are defined. $F_{m, x}$ and $F_{m, y}[\mathrm{~N}], m=\{p, q, r, s\}$ denote the $x$ and $y$-components of the joint forces, while $M_{d r v}[\mathrm{~N}-\mathrm{m}]$ is the driving torque. The $x$ and $y$-components of the shaking force acting on the supporting frame equal, respectively:

$$
F_{s h a k, x}=-\left(F_{p, x}+F_{s, x}\right) ; \quad F_{s h a k, y}=-\left(F_{p, y}+F_{s, y}\right) .
$$

The magnitude $F_{\text {shak }}[\mathrm{N}]$ of the shaking force hence equals

$$
F_{\text {shak }}=\sqrt{\left(F_{p, x}+F_{s, x}\right)^{2}+\left(F_{p, y}+F_{s, y}\right)^{2}} .
$$

The shaking moment $M_{\text {shak }}[\mathrm{N}-\mathrm{m}]$ with respect to the point $p$ equals:

$$
M_{s h a k}=-\left(M_{d r v}+F_{s, y} \cdot a_{4} \cdot \cos \phi_{4}-F_{s, x} \cdot a_{4} \cdot \sin \phi_{4}\right) .
$$

\subsection{Optimization Variables}

In order to reduce the dynamic reactions, counterweights are added to each link. The counterweights' mass parameters, indicated with an asterisk $(\cdot)^{*}$, constitute the optimization variables, and are grouped into the optimization variable vector $\mathbf{x} \in \mathbb{R}^{12}$ :

$$
\mathbf{x}=\left[\begin{array}{llllllllllll}
m_{1}^{*} & X_{1}^{*} & Y_{1}^{*} & J_{1}^{*} & m_{2}^{*} & X_{2}^{*} & Y_{2}^{*} & J_{2}^{*} & m_{3}^{*} & X_{3}^{*} & Y_{3}^{*} & J_{3}^{*}
\end{array}\right]^{T},
$$




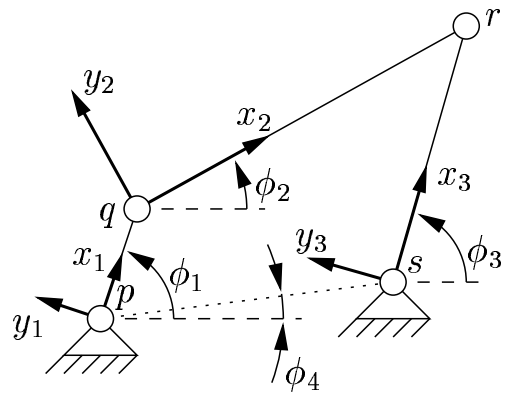

$(a)$

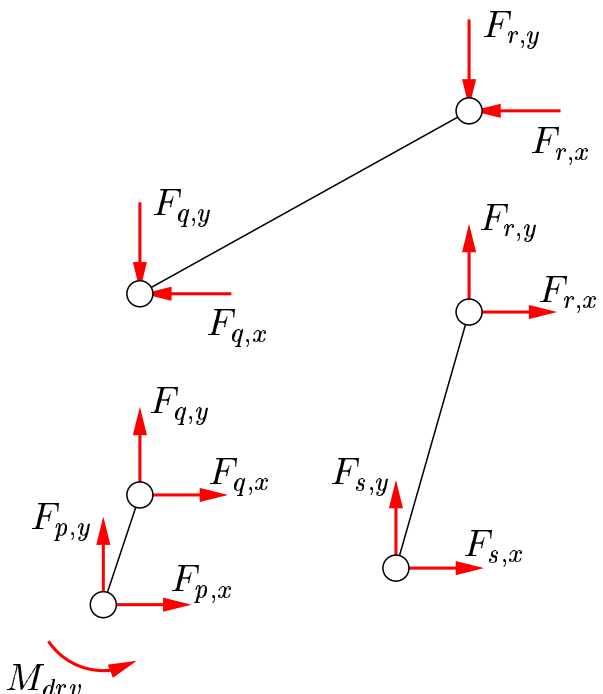

$(b)$

Figure 1: (a) Kinematic scheme of a planar crank-rocker four-bar mechanism: link 1 (crank) = $p q ;$ link 2 (coupler) $=q r ;$ link 3 (rocker) $=s r$. $(b)$ Corresponding free-body diagram.

where $(\cdot)^{T}$ denotes a matrix transpose. $m_{i}^{*}[\mathrm{~kg}]$ and $J_{i}^{*}\left[\mathrm{~kg}-\mathrm{m}^{2}\right]$ are the mass and centroidal moment of inertia of the $i$-th counterweight. For each link, the counterweight's center of gravity (COG) coordinates $X_{i}^{*}[\mathrm{~m}]$ and $Y_{i}^{*}[\mathrm{~m}], i=\{1,2,3\}$ are defined with respect to the corresponding link coordinate system $\left(x_{i}, y_{i}\right)$, defined in Fig. 1(a).

Using the counterweights' mass parameters directly as the optimization variables is the most general counterweight parametrization possible, and more general than assuming the counterweights to be circular (cylindrical) and using their radius, thickness and COG coordinates as the optimization variables.

\subsection{Objective Function}

The dimensionless balancing effect index $\alpha[-]$ is introduced in $[4,9]$ as the root-mean-square (over one period $T$ ) of the optimized dynamic reactions with respect to the root-mean-square (rms) of the original dynamic reactions, indicated with a superscript $(\cdot)^{\circ}$ :

$$
\begin{aligned}
\alpha_{f s h} & =\frac{\operatorname{rms}\left(F_{\text {shak }}\right)}{\operatorname{rms}\left(F_{\text {shak }}^{\mathrm{o}}\right)} ; \\
\alpha_{m s h} & =\frac{\operatorname{rms}\left(M_{\text {shak }}\right)}{\operatorname{rms}\left(M_{\text {shak }}^{\mathrm{o}}\right)} ;
\end{aligned}
$$




$$
\alpha_{d r v}=\frac{\operatorname{rms}\left(M_{d r v}\right)}{\operatorname{rms}\left(M_{d r v}^{o}\right)} .
$$

A classical way of optimizing the dynamic reactions is to define the objective function as a weighted combination of the three balancing effect indices:

$$
\operatorname{minimize} W_{f s h} \cdot \alpha_{f s h}+W_{m s h} \cdot \alpha_{m s h}+W_{d r v} \cdot \alpha_{d r v}
$$

where $W_{f s h}, W_{m s h}$ and $W_{d r v}$ represent (dimensionless) optimization weights. Here, however, a different approach, inspired by $[14,18]$, is adopted:

$$
\begin{aligned}
\operatorname{minimize} & \alpha_{m s h} \\
\text { subject to } & \alpha_{f s h} \leq \alpha_{f s h}^{\mathrm{M}} \\
& \alpha_{d r v} \leq \alpha_{d r v}^{\mathrm{M}} .
\end{aligned}
$$

The advantage of this approach is that the rms shaking moment is minimized while the designer directly controls, through the designer-specified upper bounds $\alpha_{d}^{\mathrm{M}}, d=\{f s h, d r v\}$, the maximum

allowed increase $\left(\alpha_{d}^{\mathrm{M}}>1, d=\{f s h, d r v\}\right)$, or the minimum wanted reduction $\left(\alpha_{d}^{\mathrm{M}}<1, d=\right.$ $\{f s h, d r v\})$ of the rms shaking force and driving torque.

\subsection{Mass Constraints}

Besides the constraints (5b)-(5c), which keep $\alpha_{f s h}$ and $\alpha_{d r v}$ under control, constraints are also required for the counterweights' mass parameters. For physical reasons, masses and moments of inertia must be positive. Moreover, bound constraints are required for the counterweight COG coordinates in order to obtain realistic counterweight configurations. Furthermore, the total counterweight mass is constrained to a maximum $m_{t o t}^{*, \mathrm{M}}[\mathrm{kg}]$. As a result, the following constraints are obtained, $i=\{1,2,3\}$ :

$$
\begin{aligned}
& m_{i}^{*} \geq 0 \\
& J_{i}^{*} \geq 0 \\
& X_{i}^{\mathrm{m}} \leq X_{i}^{*} \leq X_{i}^{\mathrm{M}} \\
& Y_{i}^{\mathrm{m}} \leq Y_{i}^{*} \leq Y_{i}^{\mathrm{M}} ; \\
& m_{1}^{*}+m_{2}^{*}+m_{3}^{*} \leq m_{t o t}^{*, \mathrm{M}},
\end{aligned}
$$

where the superscripts $(\cdot)^{\mathrm{m}}$ and $(\cdot)^{\mathrm{M}}$ denote a designer-specified lower or upper bound, respectively. These constraints allow the counterweights to combine nonzero mass with zero centroidal inertia. Such counterweights, in fact, are point masses, which are theoretically, but not practically implementable. 


\subsection{Resulting Optimization Problem}

The optimization problem with the optimization variable vector $\mathbf{x}(4)$, the objective function (5a) and the constraints (5b)-(5c) and (6a)-(6e) is nonconvex ${ }^{3}$. This is due to the fact that the balancing effect indices $\alpha_{f s h}, \alpha_{m s h}$ and $\alpha_{d r v}$ are complicated, nonconvex functions of the optimization variables. The following section reveals how this nonconvex program can be reformulated as a convex program, more specifically, an SOCP.

\section{Convex Reformulation}

The key element in deriving a convex reformulation for the optimization problem of Sec. 3.5 is to adopt another (equally general) mass parametrization, noted as $\mu_{j i}$ and defined as $i=\{1,2,3\}$ :

$$
\begin{aligned}
& \mu_{1 i}=m_{i} ; \\
& \mu_{2 i}=m_{i} \cdot X_{i} ; \\
& \mu_{3 i}=m_{i} \cdot Y_{i} ; \\
& \mu_{4 i}=J_{i}+m_{i} \cdot\left(X_{i}^{2}+Y_{i}^{2}\right) .
\end{aligned}
$$

Sections 4.1 and 4.2 discuss two essential properties of this mass parametrization. Furthermore, obtaining an SOCP requires introducing some auxiliary variables, which are formally defined in Sec. 4.3. Their use becomes clear in Sec. 4.4, which shows that the reformulated optimization problem is an SOCP.

\subsection{Mass Parametrization}

The $\mu$-parameters defined by $(7 \mathrm{a})-(7 \mathrm{~d})$ are well-known in both robotics and dynamic balancing literature and, as stressed by Haines [12], have a remarkable superposition property. That is, the $\mu$ parameters of a counterweighted link are the mere sum of the $\mu$-parameters of the unbalanced link and the counterweight (provided that they are expressed with respect to the same link coordinate system). This is trivially proven for $\mu_{1 i}, \mu_{2 i}$ and $\mu_{3 i}$ and based on the parallel axis theorem for $\mu_{4 i}$. Grouping the $\mu$-parameters of the original mechanism in $\boldsymbol{\mu}^{\circ} \in \mathbb{R}^{12}$, the counterweights' $\mu$ parameters in $\boldsymbol{\mu}^{*} \in \mathbb{R}^{12}$ and the $\mu$-parameters of the counterweighted mechanism in $\boldsymbol{\mu} \in \mathbb{R}^{12}$ yields:

$$
\boldsymbol{\mu}=\boldsymbol{\mu}^{\mathrm{o}}+\boldsymbol{\mu}^{*}
$$

\footnotetext{
${ }^{3}$ This is also the case when the radius, thickness and COG coordinates of circular counterweights are used as optimization variables.
} 
The constraints (6a)-(6e) on the added mass parameters are translated as follows to the $\mu$ parameters, $i=\{1,2,3\}$ :

$$
\begin{aligned}
& \mu_{1 i}^{*} \geq 0 \\
& \mu_{1 i}^{*} \cdot \mu_{4 i}^{*} \geq\left(\mu_{2 i}^{*}\right)^{2}+\left(\mu_{3 i}^{*}\right)^{2} ; \\
& \mu_{1 i}^{*} \cdot X_{i}^{\mathrm{m}} \leq \mu_{2 i}^{*} \leq \mu_{1 i}^{*} \cdot X_{i}^{\mathrm{M}} ; \\
& \mu_{1 i}^{*} \cdot Y_{i}^{\mathrm{m}} \leq \mu_{3 i}^{*} \leq \mu_{1 i}^{*} \cdot Y_{i}^{\mathrm{M}} ; \\
& \mu_{11}^{*}+\mu_{12}^{*}+\mu_{13}^{*} \leq m_{t o t}^{*, \mathrm{M}} .
\end{aligned}
$$

All of these constraints are linear in the $\mu$-parameters, except for (9b). In conjunction with the redundant constraint $\mu_{4 i}^{*} \geq 0,(9 \mathrm{a})-(9 \mathrm{~b})$ constitute a set of so-called hyperbolic constraints, as they describe a half hyperboloid. Using the fact [35]:

$$
\mathbf{w}^{T} \mathbf{w} \leq x y, x \geq 0, y \geq 0 \Longleftrightarrow\left\|\left[\begin{array}{c}
2 \mathbf{w} \\
x-y
\end{array}\right]\right\|_{2} \leq x+y,
$$

where $\mathbf{w}$ is a vector and $x$ and $y$ are scalars, (9a)-(9e) are equivalent to, $i=\{1,2,3\}$ :

$$
\begin{aligned}
& \left\|\left[\begin{array}{c}
2 \mu_{2 i}^{*} \\
2 \mu_{3 i}^{*} \\
\mu_{1 i}^{*}-\mu_{4 i}^{*}
\end{array}\right]\right\|_{2} \leq \mu_{1 i}^{*}+\mu_{4 i}^{*} ; \\
& \mu_{1 i}^{*} \cdot X_{i}^{\mathrm{m}} \leq \mu_{2 i}^{*} \leq \mu_{1 i}^{*} \cdot X_{i}^{\mathrm{M}} ; \\
& \mu_{1 i}^{*} \cdot Y_{i}^{\mathrm{m}} \leq \mu_{3 i}^{*} \leq \mu_{1 i}^{*} \cdot Y_{i}^{\mathrm{M}} ; \\
& \mu_{11}^{*}+\mu_{12}^{*}+\mu_{13}^{*} \leq m_{t o t}^{* \mathrm{M}} .
\end{aligned}
$$

These constraints are linear in $\mu_{j i}^{*}$ except for (10a). Comparing the latter constraint with (3) reveals that it is a second-order cone constraint in $\mu_{1 i}^{*}, \mu_{2 i}^{*}, \mu_{3 i}^{*}$ and $\mu_{4 i}^{*}$.

\subsection{Linearly Independent Vectors}

The following result is well-known in both dynamic balancing and experimental robot identification, see e.g. [36, 37]: if given time trajectories are imposed to all degrees of freedom of an (open or closed) kinematic chain of rigid bodies (in other words, if an inverse dynamic or kinetostatic analysis is carried out), then any force (dynamic reaction) $f(t)$ can be expressed as:

$$
f(t)=\mathbf{e}(t)^{T} \cdot \mathbf{p},
$$

where $\mathbf{e}(t) \in \mathbb{R}^{n}$ is a time-dependent vector, determined by the chain's kinematics, and $\mathbf{p} \in \mathbb{R}^{n}$ is a time-independent vector, of which the elements are functions of the link lengths and the 
mass parameters. Given the kinematics of the four-bar mechanism, that is, the link lengths $a_{i}$, $i=\{1,2,3,4\}$, the fixed angle $\phi_{4}$ and the time trajectory of $\phi_{1}(t)$, the following expressions for the dynamic reactions can be obtained, see e.g. $[1,11,38,39,28]$ :

$$
\begin{aligned}
F_{s h a k, x}(t) & =\mathbf{e}_{f s h x}^{T}(t) \cdot \mathbf{p}_{f s h x}=\mathbf{e}_{f s h x}^{T}(t) \cdot \mathbf{p}_{f s h} \\
F_{s h a k, y}(t) & =\mathbf{e}_{f s h y}^{T}(t) \cdot \mathbf{p}_{f s h y}=\mathbf{e}_{f s h y}^{T}(t) \cdot \mathbf{p}_{f s h} \\
M_{s h a k}(t) & =\mathbf{e}_{m s h}^{T}(t) \cdot \mathbf{p}_{m s h} \\
M_{d r v}(t) & =\mathbf{e}_{d r v}^{T}(t) \cdot \mathbf{p}_{d r v} .
\end{aligned}
$$

Explicit expressions for the elements of $\mathbf{e}_{d} \in \mathbb{R}^{n_{d}}$ and $\mathbf{p}_{d} \in \mathbb{R}^{n_{d}}, d=\{f \operatorname{sh} x, f s h y, m s h, d r v\}$ are provided in Appendix A. The variables $\mathbf{p}_{f s h x}$ and $\mathbf{p}_{f s h y}$ are treated as one variable $\mathbf{p}_{f s h}$ since $\mathbf{p}_{f s h x}=\mathbf{p}_{f s h y}$.

The elements of $\mathbf{e}_{d}$ solely depend on the mechanism's kinematics and are constructed to be linearly independent. In dynamic balancing literature, these functions are known as linearly independent vectors, a term coined in [1]. The time-independent elements of $\mathbf{p}_{d}$ are linear combinations of the $\mu$-parameters, where the coefficients depend on the (moving) link lengths only (Appendix A):

$$
\mathbf{C}_{d}\left(a_{1}, a_{2}, a_{3}\right) \cdot \boldsymbol{\mu}=\mathbf{p}_{d}, \mathbf{C}_{d} \in \mathbb{R}^{n_{d} \times 12}, d=\{f s h, m s h, d r v\}
$$

Combining this result with the linearly independent vector expressions (11a)-(11d) reveals a key aspect of using $\mu$-parameters: for given kinematics, the dynamic reactions are linear combinations of the $\mu$-parameters.

\subsection{Auxiliary Variables}

In order to come to the SOCP reformulation, an auxiliary set of variables $\mathbf{z}_{d}$ is introduced. Based on the concept of linearly independent vectors and the singular value decomposition, Appendix $\mathrm{B}$ shows that it is possible to define auxiliary variables $\mathbf{z}_{d} \in \mathbb{R}^{n_{d}}, d=\{f s h, m s h, d r v\}$ that simultaneously satisfy

$$
\begin{aligned}
& \alpha_{d}=\left\|\mathbf{z}_{d}\right\|_{2} ; \\
& \mathbf{z}_{d}=\mathbf{\Psi}_{d} \cdot \mathbf{p}_{d},
\end{aligned}
$$

where the matrices $\boldsymbol{\Psi}_{d} \in \mathbb{R}^{n_{d} \times n_{d}}$ only depend on the mechanism kinematics.

Furthermore, an auxiliary variable $q$ is introduced to transform the original objective function (5a) into an objective function that is linear in the optimization variables, using the common trick 
already explained in Sec. 2:

$$
\operatorname{minimize} \quad \alpha_{m s h}
$$

is equivalent to

$$
\begin{aligned}
\text { minimize } & q \\
\text { subject to } & \alpha_{m s h} \leq q .
\end{aligned}
$$

\subsection{Resulting SOCP}

This section shows that, based on the variables and constraints defined in Secs. 4.1, 4.2 and 4.3, the original nonlinear optimization problem of Sec. 3 transforms into an SOCP. First, all optimization variables are grouped into the new optimization variable vector $\mathbf{x}$ :

$$
\mathbf{x}=\left[\begin{array}{lllllllll}
\left(\boldsymbol{\mu}^{*}\right)^{T} & \boldsymbol{\mu}^{T} & \mathbf{p}_{f s h}^{T} & \mathbf{p}_{m s h}^{T} & \mathbf{p}_{d r v}^{T} & \mathbf{z}_{f s h}^{T} & \mathbf{z}_{m s h}^{T} & \mathbf{z}_{d r v}^{T} & q
\end{array}\right]^{T} .
$$

$\mathbf{x} \in \mathbb{R}^{49}$ if the input crank speed $\dot{\phi}_{1}$ is constant and $\mathbf{x} \in \mathbb{R}^{53}$ if it is fluctuating. The objective is simply (14a):

$$
\text { minimize } q \text {. }
$$

The minimum is to be sought subject to the following constraints:

1. linear equality constraints (8), due to the superposition of the original and counterweight $\mu$-parameters, which converts $\boldsymbol{\mu}^{*}$ to $\boldsymbol{\mu}$;

2. linear equality constraints, due to the linear conversion (12) from $\boldsymbol{\mu}$ to $\mathbf{p}_{d}, d=\{f s h, m s h, d r v\}$;

3. linear equality constraints, due to the linear conversion (13b) from $\mathbf{p}_{d}$ to $\mathbf{z}_{d}, d=\{f s h, m s h, d r v\}$;

4. linear inequality constraints (10b)-(10d) in the counterweight $\mu^{*}$-parameters, $i=\{1,2,3\}$;

5. second-order cone constraints (10a) in the counterweight $\mu^{*}$-parameters, $i=\{1,2,3\}$;

6. nonlinear constraints (5b)-(5c), (14b). Given (13a), they are equivalent to the following second-order cone constraints in $q$ and $\mathbf{z}_{d}, d=\{f s h, m s h, d r v\}$ :

$$
\begin{aligned}
\left\|\mathbf{z}_{f s h}\right\|_{2} & \leq \alpha_{f s h}^{\mathrm{M}} \\
\left\|\mathbf{z}_{d r v}\right\|_{2} & \leq \alpha_{d r v}^{\mathrm{M}} \\
\left\|\mathbf{z}_{m s h}\right\|_{2} & \leq q .
\end{aligned}
$$




\begin{tabular}{|c||l|l|l||l|l|l|}
\cline { 2 - 7 } \multicolumn{1}{c||}{} & \multicolumn{3}{c||}{ Example 1 [18, 19] } & \multicolumn{3}{c|}{ Example 2 [14] } \\
\cline { 2 - 7 } & $i=1$ & $i=2$ & $i=3$ & $i=1$ & $i=2$ & $i=3$ \\
\hline \hline$a_{i}[\mathrm{~m}]$ & 0.0508 & 0.1524 & 0.0762 & 0.36 & 1.09 & 0.54 \\
\hline$m_{i}[\mathrm{~kg}]$ & 0.0894 & 0.2394 & 0.1215 & 0.21 & 0.55 & 0.24 \\
\hline$X_{i}[\mathrm{~m}]$ & 0.0254 & 0.0762 & 0.0381 & 0.1800 & 0.5574 & 0.2700 \\
\hline$Y_{i}[\mathrm{~m}]$ & 0.0 & 0.0102 & 0.0 & 0.0 & 0.1603 & 0.0 \\
\hline$J_{i}\left[\mathrm{~kg}-\mathrm{m}^{2}\right]$ & 0.0000198 & 0.0006792 & 0.0002198 & 0.002 & 0.030 & 0.006 \\
\hline \hline$a_{4}[\mathrm{~m}]$ & \multicolumn{3}{|c||c|c|c|}{1.0} \\
\hline$\phi_{4}[\mathrm{~m}]$ & \multicolumn{3}{|||}{0.1397} & 0.0 & \multicolumn{3}{c}{0} \\
\hline \hline
\end{tabular}

Table 1: Crank-rocker four-bar examples: numerical link parameters.

The resulting optimization problem has a linear objective function that is minimized subject to linear equality constraints, linear inequality constraints and second-order cone constraints. It is hence a second-order cone program. This convex program is equivalent to the original, nonconvex optimization problem.

\section{$5 \quad$ Numerical Example 1 - Design Procedure}

By applying the SOCP to a numerical example, previously studied in $[18,19]$, this section introduces a three-step design procedure that allows the designer to make a well-balanced choice in the difficult trade-off problem that constitutes counterweight balancing. First, the raw optimization results need to be generated (Sec. 5.1). Second, the raw results are converted into trade-off curves, which constitute the heart of the presented method (Sec. 5.2). Finally, the ultimate counterweights obtained, which are point-masses, need to be converted into practical counterweights (Sec. 5.3).

\subsection{Raw Optimization Results}

The SOCP is applied to a planar crank-rocker four-bar linkage previously studied in $[18,19]$. Table 1 defines the linkage parameters. The crank revolves with a constant speed of $500 \mathrm{rpm}$. Given that this purely inertial mechanism is balanced by mass addition only, all balancing results (in terms of the balancing indices $\alpha_{d}$ ) are independent of the applied (constant) drive speed.

The following values are adopted for the upper and lower bounds on the COG coordinates used 
in (10b)-(10c), $i=\{1,2,3\}$ :

$$
\begin{aligned}
& X_{i}^{\mathrm{m}}=-\frac{a_{i}}{2} ; X_{i}^{\mathrm{M}}=\frac{3 a_{i}}{2} \\
& Y_{i}^{\mathrm{m}}=-\frac{a_{i}}{2} ; Y_{i}^{\mathrm{M}}=\frac{a_{i}}{2} .
\end{aligned}
$$

Using the dimensionless parameter $\eta$, the upper limit $m_{\text {tot }}^{*, \mathrm{M}}[\mathrm{kg}]$ on the total counterweight mass is expressed as a fraction of the total mass $m_{t o t}^{o}=0.4503 \mathrm{~kg}$ of the unbalanced links:

$$
m_{t o t}^{*, \mathrm{M}}=\eta \cdot m_{t o t}^{o}
$$

Four different values of $\eta$ are considered $\eta=\{0.50,0.75,1.00,2.00\}, 101$ equidistant values of $\alpha_{f s h}^{\mathrm{M}}$ between 0 and 1 , as well as 76 equidistant values of $\alpha_{d r v}^{\mathrm{M}}$ between 0.5 and 1.25. This implies that, for each of the four values of $\eta$, the SOCP needs to be solved 7676 times, which, to give an idea of the great numerical efficiency, takes 18790 sec on a PentiumIV@2.8GHz processor, or an average of $0.612 \mathrm{sec}$ per optimization. The optimization problem is implemented in Matlab, using Yalmip, a Matlab toolbox for modeling optimization problems independent of the numerical solver [40]. The SOCP numerical solver used here is SeDuMi, a dedicated software package for optimization problems over symmetric cones [41].

A very useful feature of convex programs, like the SOCP at hand, is the way in which infeasible problems are handled. If the considered $\left(\eta, \alpha_{f s h}^{\mathrm{M}}, \alpha_{d r v}^{\mathrm{M}}\right)$ combination is such that it is impossible to comply simultaneously with all constraints, SeDuMi does not crash, but yields a so-called certificate of infeasibility, which is a numerical proof that the problem is infeasible. For each of the considered $\eta$-values, the following number of the $7676\left(\alpha_{f s h}^{\mathrm{M}}, \alpha_{d r v}^{\mathrm{M}}\right)$ combinations yields infeasible problems: $6650(\eta=0.50) ; 6154(\eta=0.75) ; 5608(\eta=1.00) ; 3523(\eta=2.00)$.

For all feasible problems, the resulting optimum is characterized by a total counterweight mass equal to its upper limit $m_{\text {tot }}^{*, \mathrm{M}}$, realized as counterweights with zero centroidal inertia $J_{i}^{*}$, that is, point-mass counterweights. For most feasible problems, $\alpha_{f s h}=\alpha_{f s h}^{\mathrm{M}}$ and $\alpha_{d r v}=\alpha_{d r v}^{\mathrm{M}}$, although some problems have either $\alpha_{f s h}<\alpha_{f s h}^{\mathrm{M}}$ or $\alpha_{d r v}<\alpha_{d r v}^{\mathrm{M}}$.

The raw optimization results are displayed in Fig. 2 as contour plots of $\alpha_{m s h}$ as a function of $\alpha_{f s h}$ and $\alpha_{d r v}$, for the four selected $\eta$-values. The white region marked $I$ is the infeasible region, which corresponds to $\left(\alpha_{f s h}^{\mathrm{M}}, \alpha_{d r v}^{\mathrm{M}}\right)$ pairs that yield infeasible problems. Quite logically, the size of the infeasible area $I$ shrinks if the constraint on the total counterweight mass is relaxed (by increasing $\eta$ ). The white region marked $F$ denotes the part of the feasible region for which data is not available. That is, the corresponding $\left(\alpha_{f s h}, \alpha_{d r v}\right)$ pair can be realized using point masses that satisfy all constraints, but such pairs did simply not result from the chosen range for $\alpha_{f s h}^{\mathrm{M}}$ 
(a)

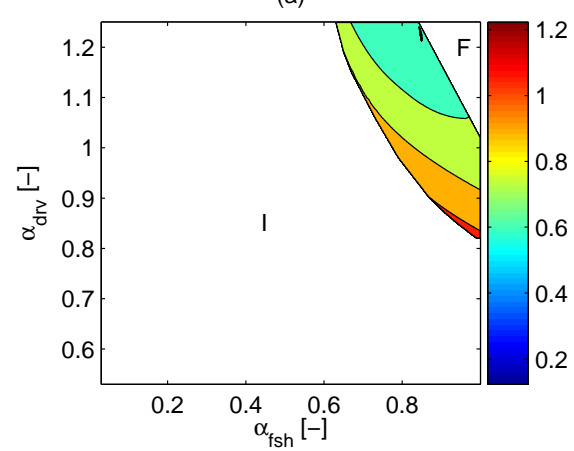

(c)

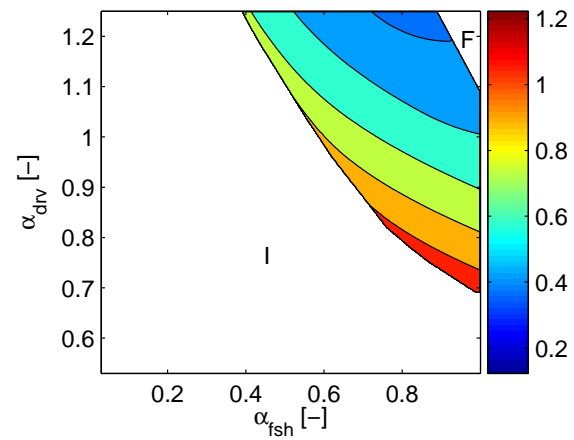

(b)

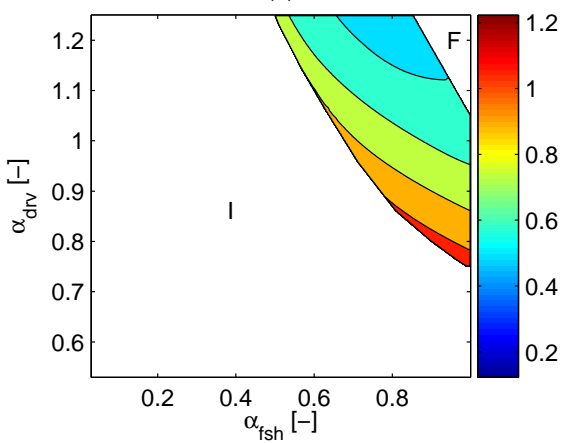

(d)

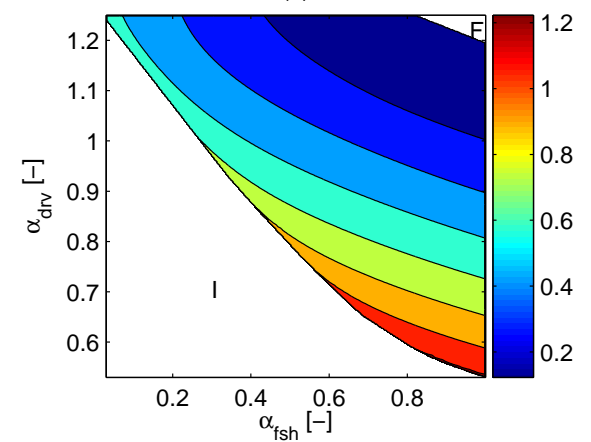

Figure 2: Contour plots of $\alpha_{m s h}[-]$ as a function of $\alpha_{f s h}[-]$ and $\alpha_{d r v}[-]$ for $\eta$ equal to $\{0.50(a), 0.75(b), 1.00(c), 2.00(d)\}$. The white region marked $I$ is the infeasible region. The white region marked $F$ denotes the part of the feasible region for which data is not available. In each figure, the value of $\alpha_{m s h}$ increases from the top to the bottom of the figure.

and $\alpha_{d r v}^{\mathrm{M}}\left({ }^{4}\right)$.

Fig. 2 shows that for the chosen parameter range full force balance $\left(\alpha_{f s h}=0\right)$ is not possible. Additional numerical experiments show that full force balance is possible by allowing (while keeping $\left.\alpha_{d r v}^{\mathrm{M}}=1.2\right)$ a total counterweight mass greater than $\eta=2.2$ times the original mechanism mass. If, on the other hand, the upper limit (15b) on $\alpha_{d r v}$ is dropped, the minimum required counterweight mass resulting in full force balance equals $\eta=1.54$ times the original mass. Such large counterweight masses for obtaining full force balance are not exceptional. Full force balance of the crank-rocker four-bars considered in [1] and [9], for instance, requires total counterweight masses equal to $\eta=1.47$ and $\eta=1.67$ times the original mechanism mass, respectively.

Fig. 2 also shows that zero driving torque $\left(\alpha_{d r v}=0\right)$ is not possible. This is not a surprise: even if the amount of counterweight mass increased to infinity, zero driving torque could not be obtained:

\footnotetext{
${ }^{4}$ This is due to the aforementioned presence of optima in which the actually obtained $\alpha_{f s h}$ or $\alpha_{d r v}$ are lower than the imposed upper limits $\alpha_{f s h}^{\mathrm{M}}$ or $\alpha_{d r v}^{\mathrm{M}}$.
} 
only a massless four-bar can have zero driving torque [11].

\subsection{Trade-off Curves}

In order to further interpret the results displayed in Fig. 2, cuts are made in each of the Figs. $2(a)-(b)-(c)-(d)$ with cutting planes along the lines $\alpha_{d r v}=1.2$ and $\alpha_{f s h}=0.66$, which gives rise to four lines in Fig. $3(a)$ and $(b)$, respectively ${ }^{5}$. Fig. 3(b) shows that $\alpha_{m s h}$ is very sensitive to $\alpha_{d r v}$ : for instance for $\eta=2.00$, allowing a $25 \%$ increase of $\alpha_{d r v}$ from 0.8 to 1.0 results in almost twice as large a $46 \%$ decrease of $\alpha_{m s h}$ from 0.76 to 0.41 . This plot therefore suggests that $\alpha_{d r v}$ should be chosen as large as possible, since the corresponding reduction in $\alpha_{m s h}$ is big. This explains why Fig. 3(a) is plotted for $\alpha_{d r v}=1.2$. This figure shows that for higher values of $\alpha_{f s h}$, the decrease in shaking moment, by allowing a larger shaking force is small. It therefore seems appropriate to choose $\alpha_{f s h}$ between 0.6 and 0.8 (for instance, $\alpha_{f s h}=0.66$ ), that is, in the part of the trade-off curve in between the steeper and the flatter parts.

In order to choose an appropriate $\eta$-value, new optimizations are carried out with $\left(\alpha_{f s h}=\right.$ $\left.0.66, \alpha_{d r v}=1.20\right)$ and 31 equidistant $\eta$-values ranging between 0.50 and $2.00\left({ }^{6}\right)$. Figure $4(a)$ shows that $\alpha_{m s h}$ is more sensitive to $\eta$ for low values of $\eta$. Figure $4(c)$ indicates that for $\eta \leq 0.80$, no counterweight is attached to the coupler. Based on these plots, $\eta=0.80$, yielding $\alpha_{m s h}=0.60$ seems a good compromise: featuring no coupler counterweight, it results in a cheaper counterweight arrangement and moreover lies just past the low $\eta$-values where $\alpha_{m s h}$ is more sensitive to $\eta$. Furthermore, $\eta=0.80$ is very reasonable as compared to the previously mentioned $\eta=1.47$ and $\eta=1.67$-values of [1] and [9].

For the selected combination $\left(\eta=0.80, \alpha_{f s h}=0.66, \alpha_{d r v}=1.20\right)$, the resulting crank and rocker counterweight parameters are given in the $P M$ column of Table 2. Besides the zero lower limit on the centroidal inertia, also the lower limit on the $\mathrm{X}$-coordinate of the first counterweight is active. The nonzero Y-coordinates indicate that neither of the counterweights is inline.

The corresponding balancing effect indices are given in Table 3 . The balancing indices $\alpha_{d}, d=$ $\{f p, f q, f r, f s\}$ are defined in a similar manner as $\alpha_{d}, d=\{f s h, m s h, d r v\}$, but for the magnitude of the forces in the joints $p, q, r$ and $s$. The rms of all joint forces increases, but in a reasonable

\footnotetext{
${ }^{5}$ Note that the vertical line $\alpha_{f s h}=0.66$ in Fig. $3(a)$ and the vertical line $\alpha_{d r v}=1.20$ in Fig. $3(b)$ intersect corresponding trade-off lines at equal $\alpha_{m s h}$ values.

${ }^{6}$ Not shown in Fig. $4(a)$ is that, for $\eta \rightarrow \infty, \alpha_{m s h} \rightarrow 0.07$, illustrating that for a general crank-rocker four-bar as the one considered here, shaking moment balance cannot be obtained by only adding counterweights. If shaking force balance is imposed $\left(\alpha_{f s h}^{\mathrm{M}}=0\right)$, while dropping the upper limit $(15 \mathrm{~b})$ on $\alpha_{d r v}, \eta \rightarrow \infty$ results in $\alpha_{m s h} \rightarrow 0.16$.
} 

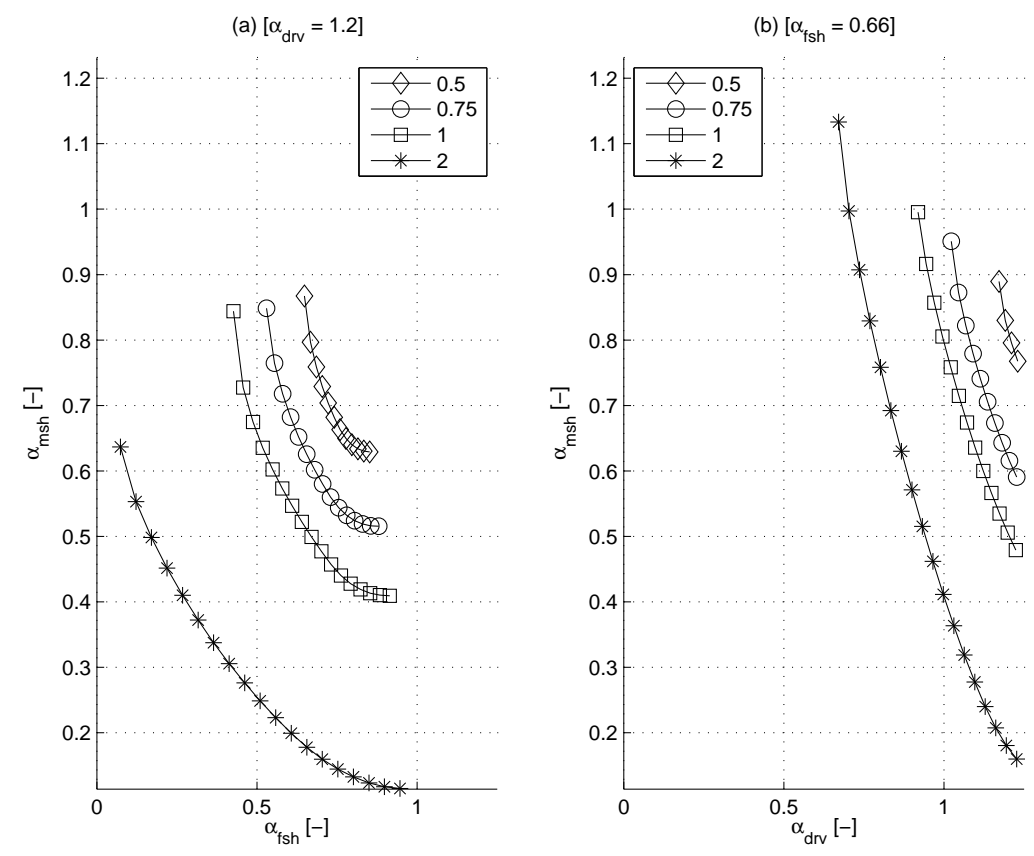

Figure 3: (a) $\alpha_{m s h}$ as a function of $\alpha_{f s h}$ for a fixed value $\alpha_{d r v}=1.20$. The four lines mark four different values of $\eta=\{0.50,0.75,1.00,2.00\}$. (b) $\alpha_{m s h}$ as a function of $\alpha_{d r v}$ for a fixed value $\alpha_{f s h}=0.66$.
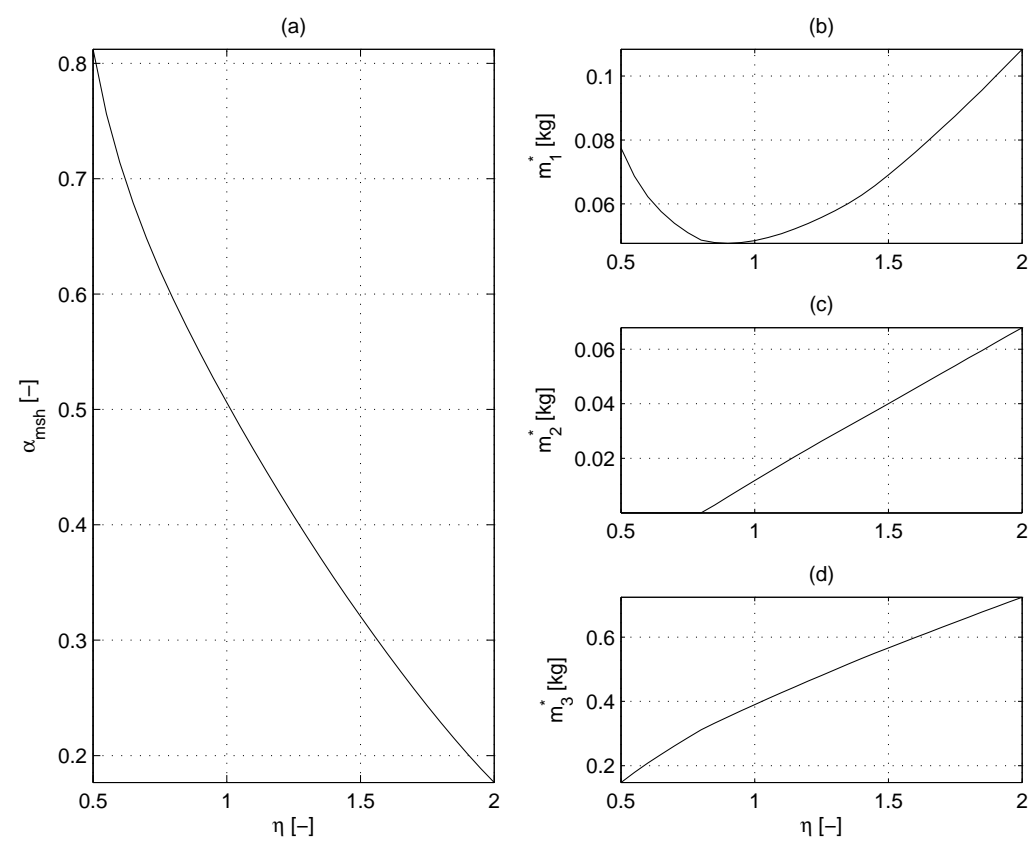

(c)

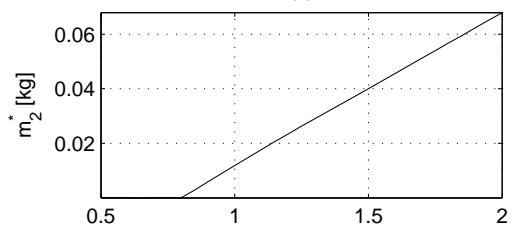

(d)

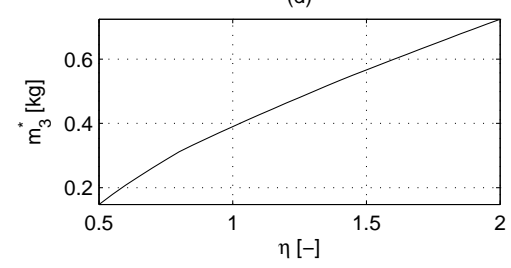

Figure 4: Ultimate balancing limits as a function of $\eta$ for $\left(\alpha_{f s h}=0.66, \alpha_{d r v}=1.20\right) .(a) \alpha_{m s h} ;(b)$ mass of crank counterweight; $(c)$ mass of coupler counterweight; $(d)$ mass of rocker counterweight. 


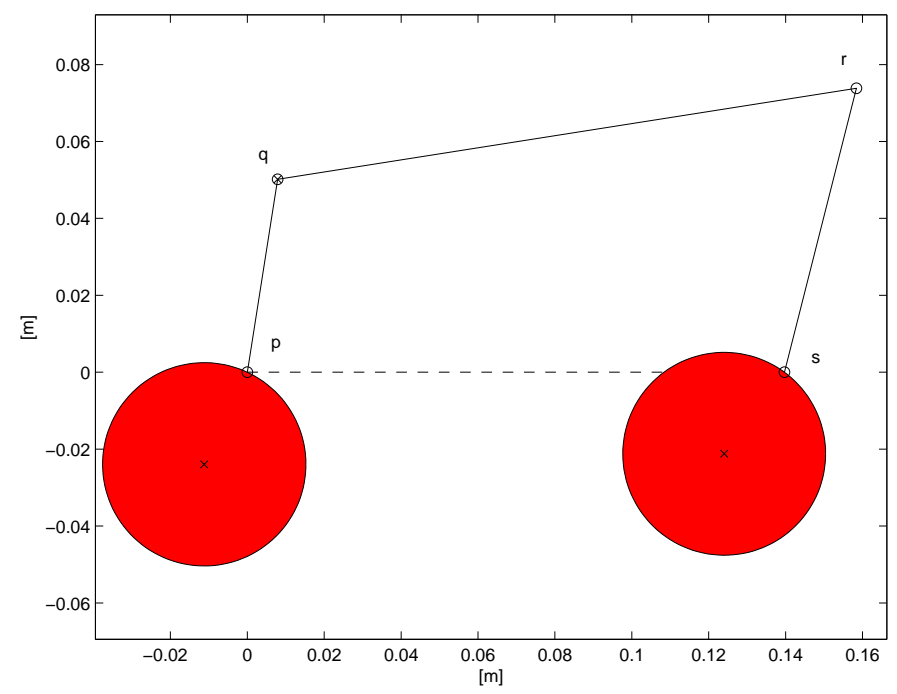

Figure 5: Counterweighted crank-rocker four-bar, after transforming the point-mass counterweights for $\left(\eta=0.80, \alpha_{f s h}=0.66, \alpha_{d r v}=1.20\right)$ to circular, minimum inertia counterweights. The counterweight radiuses equal: $R_{c, 1}^{*}=26.45$ and $R_{c, 3}^{*}=26.37 \mathrm{~mm}$. The counterweight thicknesses equal $t_{c, 1}^{*}=2.83$ and $t_{c, 3}^{*}=18.20 \mathrm{~mm}$.

\begin{tabular}{||c|r|r|r|r||}
\cline { 2 - 5 } \multicolumn{1}{c|}{} & \multicolumn{1}{c|}{$L$} & \multicolumn{1}{c|}{$\mathrm{PM}$} & \multicolumn{1}{c|}{$\mathrm{CC}$} & \multicolumn{1}{c||}{$U$} \\
\hline \hline$m_{1}^{*}[\mathrm{~g}]$ & 0.0 & 48.7 & 48.7 & $\ldots$ \\
$m_{3}^{*}[\mathrm{~g}]$ & 0.0 & 311.6 & 311.6 & $\ldots$ \\
\hline$X_{1}^{*}[\mathrm{~mm}]$ & -25.4 & -25.4 & -25.4 & 76.2 \\
$X_{3}^{*}[\mathrm{~mm}]$ & -38.1 & -24.4 & -24.4 & 114.3 \\
\hline$Y_{1}^{*}[\mathrm{~mm}]$ & -25.4 & 7.4 & 7.4 & 25.4 \\
$Y_{3}^{*}[\mathrm{~mm}]$ & -38.1 & 10.0 & 10.0 & 38.1 \\
\hline$J_{1}^{*}\left[\mathrm{~kg}-\mathrm{mm}^{2}\right]$ & 0.0 & 0.0 & 17.0 & $\ldots$ \\
$J_{3}^{*}\left[\mathrm{~kg}-\mathrm{mm}^{2}\right]$ & 0.0 & 0.0 & 108.3 & $\ldots$ \\
\hline \hline
\end{tabular}

Table 2: Point-mass $(\mathrm{PM})$ versus circular $(\mathrm{CC})$ counterweights: comparison of counterweight parameters. No counterweight is attached to the coupler. The columns $L$ and $U$ give the corresponding lower and upper limits, respectively. 
manner: between 14 and 24 percent.

\subsection{Practical Counterweights}

The obtained ultimate counterweights are point masses and hence not implementable in practice. For practical implementation a particular counterweight shape has to be chosen ${ }^{7}$, which inevitably results in nonzero counterweight centroidal inertia, and hence worse balancing results. Both counterweights are implemented here as minimum inertia counterweights [25, 26], which are classically used in balancing four-bar mechanisms. Such counterweights realize given mass-distance products $\mu_{2 i}^{*}$ and $\mu_{3 i}^{*}$ with minimum inertia with respect to the origin $o_{i}$ of the link coordinate system in which the mass-distance products are expressed. A minimum inertia counterweight is circular, with $o_{i}$ lying on its circumference.

The circular counterweights are parametrized by their COG location $\left(X_{c, i}^{*}, Y_{c, i}^{*}\right)$, radius $R_{c, i}^{*}[\mathrm{~m}]$ and thickness $t_{c, i}^{*}[\mathrm{~m}](i=1,3)$. The circular counterweight parameters are chosen such that not only the mass-distance products $m_{c, i}^{*} X_{c, i}^{*}$ and $m_{c, i}^{*} Y_{c, i}^{*}$ are identical to the mass-distance products of the point-mass counterweights, but also the mass $m_{c, i}^{*}$ itself. Hence the COG-locations of the point-mass counterweights and the circular counterweights must coincide:

$$
X_{c, i}^{*}=X_{i}^{*} ; Y_{c, i}^{*}=Y_{i}^{*}
$$

The radius $R_{c, i}^{*}$ is such that the origin of the link coordinate system lies on the counterweight's circumference:

$$
R_{c, i}^{*}=\sqrt{\left(X_{c, i}^{*}\right)^{2}+\left(Y_{c, i}^{*}\right)^{2}}
$$

Since the point-mass counterweights and the circular counterweights must have the same mass, the thickness is determined as:

$$
t_{c, i}^{*}=\frac{m_{i}^{*}}{\pi \cdot\left(R_{c, i}^{*}\right)^{2} \cdot \rho_{c}},
$$

where $\rho_{c}\left[\mathrm{~kg} / \mathrm{m}^{3}\right]$ denotes the mass density of the counterweight. Based on the numerical data of Table 2, the steel counterweights $\left(\rho_{c}=7833 \mathrm{~kg} / \mathrm{m}^{3}\right)$ shown in Fig. 5 are obtained. The counterweight thicknesses equal 0.45 (crank) or 2.87 (rocker) times the original link thickness of $6.35 \mathrm{~mm}$.

Table 3 focuses on the effect on the balancing effect indices of using circular instead of point-mass counterweights. The only difference between these counterweights is the nonzero centroidal inertia

\footnotetext{
${ }^{7}$ In fact, the counterweight shape does not matter for the crank, since it revolves at constant speed, resulting in no influence of the counterweight inertia on the balancing effect indices.
} 


\begin{tabular}{c|c|c||c|c|c||}
\cline { 2 - 5 } \multicolumn{1}{c|}{} & $\mathrm{PM}$ & $\mathrm{CC}$ & & $\mathrm{PM}$ & $\mathrm{CC}$ \\
\hline \hline$\alpha_{f s h}[-]$ & 0.66 & 0.66 & $\alpha_{f p}[-]$ & 1.14 & 1.22 \\
$\alpha_{m s h}[-]$ & 0.60 & 0.65 & $\alpha_{f q}[-]$ & 1.18 & 1.27 \\
$\alpha_{d r v}[-]$ & 1.20 & 1.30 & $\alpha_{f r}[-]$ & 1.24 & 1.37 \\
$\ldots$ & $\ldots$ & $\ldots$ & $\alpha_{f s}[-]$ & 1.21 & 1.33 \\
\hline \hline
\end{tabular}

Table 3: Point-mass (PM) versus circular (CC) counterweights: comparison of balancing effect indices.

of the former (see Table 2). Hence, $\alpha_{f s h}$ is not affected, since it is independent of the counterweights' inertia. The nonzero inertia of the circular counterweights causes all other balancing effect indices to increase, but this increase of between 7 and $11 \%$ is judged to be surprisingly low. The final result, using circular counterweights, is a simultaneous one-third reduction of the rms shaking force and shaking moment, at the cost of (i) an increase of the rms driving torque and joint forces between 22 and $33 \%$, and (ii) two counterweights with a total mass that is $\eta=0.80$ times the original mechanism's mass.

The design proposed here has by no means 'absolute' value: it just serves as a simple example of how the optimization framework can be used to balance a mechanism. For instance, the parameters $\left(\eta, \alpha_{f s h}^{\mathrm{M}}, \alpha_{d r v}^{\mathrm{M}}\right)$ have been selected purely based on the trade-off Figs. 3 and 4 . In a practical setting many more considerations have an impact, one of the more important ones being the validity of the assumed rigid body model after adding so much mass to the mechanism. The authors however feel that the framework developed here can be of tremendous help in assessing the many design tradeoffs by providing a way of computing globally optimal counterweights in a reliable and extremely fast manner.

\section{$6 \quad$ Numerical Example 2 - Benchmarking}

This section illustrates the versatility and power of the proposed framework, by (i) reformulating Sadler and Mayne's [14] variant of ultimate CWB as a convex problem, and (ii) showing that the numerical results obtained are at least as good as the ones presented in [14]. 


\subsection{Sadler and Mayne's Problem Formulation}

Table 1 summarizes the numerical parameters of the planar crank-rocker four-bar linkage studied by Sadler and Mayne. The crank revolves with a constant speed of $1 \mathrm{rad} / \mathrm{s}$. Sadler and Mayne optimize the mass and COG location of point-mass counterweights $\left(J_{i}^{*}=0, i=\{1,2,3\}\right)$ connected to the crank and rocker (no coupler counterweight is allowed: $m_{2}=0$ ). The optimization variable vector $\mathbf{x} \in \mathbb{R}^{12}$ hence equals:

$$
\mathbf{x}=\left[\begin{array}{llllllllllll}
m_{1}^{*} & X_{1}^{*} & Y_{1}^{*} & J_{1}^{*} & m_{2}^{*} & X_{2}^{*} & Y_{2}^{*} & J_{2}^{*} & m_{3}^{*} & X_{3}^{*} & Y_{3}^{*} & J_{3}^{*}
\end{array}\right]^{T} .
$$

Sadler and Mayne determine $\mathbf{x}$ as the solution of the following optimization problem:

$$
\begin{array}{ll}
\operatorname{minimize}_{x} & \max \left(\mathbf{F}_{\text {shak }}\right) \\
\text { subject to } & \max \left(\left|\mathbf{M}_{\text {shak }}\right|\right) \leq M_{\text {shak }}^{\mathrm{M}} \\
& m_{1}^{*}+m_{3}^{*} \leq 1, m_{2}^{*}=0 \\
& m_{i}^{*} \geq 0, i=\{1,3\} \\
& J_{1}^{*}=0, i=\{1,2,3\},
\end{array}
$$

where $\mathbf{F}_{s h a k}$ and $\mathbf{M}_{\text {shak }} \in \mathbb{R}^{N}$ are determined according to (26) and the integer $N=T / T_{s}$ relates the sample period $T_{s}$ to one period of motion $T$. Given the equality constraints $m_{2}^{*}=0$ and (17e), only six optimization variables are left for optimization, that is, $m_{i}^{*}, X_{i}^{*}$ and $Y_{i}^{*}, i=\{1,3\}$. These are determined so as to minimize (using a maximum amount of $1 \mathrm{~kg}$ of counterweight point-mass) the maximum (over one period of motion $T$ ) of the shaking force, subject to the constraint that the maximum absolute value of the shaking moment ${ }^{8}$ be smaller than some user-defined upper limit $M_{\text {shak }}^{\mathrm{M}}[\mathrm{N}-\mathrm{m}]$.

\subsection{Convex Reformulation}

Like the optimization problem discussed in Sec. 3.5, the optimization problem (17a)-(17e) is nonconvex in the optimization variables (16). In order to obtain a convex program, several steps are required. Firstly, we observe that (17e) can be relaxed to

$$
J_{1}^{*} \geq 0, i=\{1,2,3\},
$$

\footnotetext{
${ }^{8}$ Sadler and Mayne chose the reference point for the shaking moment calculation halfway between the fixed pivots $p$ and $s$, as opposed to the example of Section 5 , in which the reference point coincides with $p$.
} 
since this constraint is expected ${ }^{9}$ to be always active (implying $J_{i}^{*}=0$ ) in the obtained optimum. Second, a nonlinear change of variables is carried out according to (7a)-(7d). This implies replacing (17c), (17d) and (18) by the equivalent constraints (see Sec. 4.1)

$$
\begin{gathered}
\mu_{11}^{*}+\mu_{13}^{*} \leq 1 ; \mu_{12}=0 ; \\
\left\|\left[\begin{array}{c}
2 \mu_{2 i}^{*} \\
2 \mu_{3 i}^{*} \\
\mu_{1 i}^{*}-\mu_{4 i}^{*}
\end{array}\right]\right\|_{2} \leq \mu_{1 i}^{*}+\mu_{4 i}^{*}, i=\{1,2,3\}
\end{gathered}
$$

as well as introducing (8) to establish the relation between $\boldsymbol{\mu}$ and $\boldsymbol{\mu}^{*}$.

Thirdly, we observe that $(17 \mathrm{a})-(17 \mathrm{~b})$ is equivalent to

$$
\begin{aligned}
\operatorname{minimize}_{[x ; q]} & q \\
\text { subject to } & -M_{s h a k}^{\mathrm{M}} \leq M_{s h a k}\left(k \cdot T_{s}\right) \leq M_{s h a k}^{\mathrm{M}}, k=1 \ldots N \\
& F_{\text {shak }}\left(k \cdot T_{s}\right) \leq q, k=1 \ldots N,
\end{aligned}
$$

where $q$ is an additional, auxiliary variable. The equivalence between (17a)-(17b) and (20a)-(20c) follows from the earlier discussed equivalence between (1) and (2a)-(2b), and the property that

$$
|x| \leq x^{\mathrm{M}} \Leftrightarrow-x^{\mathrm{M}} \leq x \leq x^{\mathrm{M}}
$$

Finally, we exploit (11a)-(11c) to replace (20b)-(20c) by the equivalent constraints

$$
\begin{aligned}
& -M_{s h a k}^{\mathrm{M}} \leq \mathbf{e}_{m s h}\left(k \cdot T_{s}\right) \cdot \mathbf{p}_{m s h} \leq M_{s h a k}^{\mathrm{M}}, k=1 \ldots N \\
& \left\|\left[\begin{array}{l}
\mathbf{e}_{f s h x}\left(k \cdot T_{s}\right) \cdot \mathbf{p}_{f s h} \\
\mathbf{e}_{f s h y}\left(k \cdot T_{s}\right) \cdot \mathbf{p}_{f s h}
\end{array}\right]\right\|_{2} \leq q, k=1 \ldots N
\end{aligned}
$$

where the relation between $\boldsymbol{\mu}$ and $\mathbf{p}_{f s h}$ and $\mathbf{p}_{m s h}$ is given by (12).

\subsection{Resulting SOCP}

This section shows that, based on the variables and constraints defined in the previous section, the original nonlinear optimization problem (17a)-(17e) transforms into an SOCP. First, all optimization variables are grouped into the new optimization variable vector $\mathbf{x}$ :

$$
\mathbf{x}=\left[\begin{array}{lllll}
\left(\boldsymbol{\mu}^{*}\right)^{T} & \boldsymbol{\mu}^{T} & \mathbf{p}_{f s h}^{T} & \mathbf{p}_{m s h}^{T} & q
\end{array}\right]^{T} .
$$

\footnotetext{
${ }^{9}$ This has to be checked always in the obtained optimum: if $J_{i}^{*}=0, \forall i$, relaxing (17d) to (18) has had no resulting effect on the obtained optimum. If, on the other hand, $J_{i}^{*}>0$ for some $i$, the obtained optimal value provides a lower bound for the 'true' optimal value.
} 
$\mathbf{x} \in \mathbb{R}^{36}$ if the input crank speed $\dot{\phi}_{1}$ is constant and $\mathbf{x} \in \mathbb{R}^{37}$ if it is fluctuating. The objective is simply (20a):

$$
\text { minimize } q \text {. }
$$

The minimum is to be sought subject to the following constraints:

1. linear equality constraints (8), due to the superposition of the original and counterweight $\mu$-parameters, which converts $\boldsymbol{\mu}^{*}$ to $\boldsymbol{\mu}$;

2. linear equality constraints, due to the linear conversion (12) from $\boldsymbol{\mu}$ to $\mathbf{p}_{d}, d=\{f s h, m s h\}$;

3. linear (in)equality constraints (19a) in the counterweight $\mu^{*}$-parameters;

4. linear inequality constraints (21a) in $\mathbf{p}_{m s h}$;

5. second-order cone constraints (19b) in the counterweight $\mu^{*}$-parameters;

6. second-order cone constraints (21b) in $\mathbf{p}_{f s h}$ and $q$.

This optimization problem has a linear objective function that is minimized subject to linear equality constraints, linear inequality constraints and second-order cone constraints. It is hence a second-order cone program. This convex program is a relaxation of the original, nonconvex optimization problem, since it is based on relaxing (17e) to (18). In practice, however, the optimum will always be characterized by $J_{i}^{*}=0, \forall i$, implying 'practical' equivalence of the second-order cone program and the original, nonconvex program.

\subsection{Numerical Results}

Sadler and Mayne solve the nonconvex (and nondifferentiable) optimization problem (17a)-(17e) through a penalty function approach, that is, as a sequence of unconstrained optimization problems ${ }^{10}$. They provide four optimal points corresponding to four values of $M_{s h a k}^{\mathrm{M}}: M_{s h a k}^{\mathrm{M}}=$ $\{0.3509,0.2247,0.1219,0.0853\} \mathrm{N}-\mathrm{m}$, which are summarized in Table 4 , together with four results for the (relaxed) SOCP, corresponding to the same values of $M_{\text {shak }}^{\mathrm{M}}$.

The (relaxed) SOCP is solved using SeDuMi, for $M_{s h a k}^{\mathrm{M}}$ equal to the four values considered by Sadler and Mayne. In order to numerically solve the program, additional upper and lower bounds (10b)(10c) on the counterweight COG coordinates have to be taken into account since otherwise the

\footnotetext{
${ }^{10}$ Each of the unconstrained optimization problems is solved using the Davidon-Fletcher-Powell method, a socalled quasi-Newton method for unconstrained optimization.
} 
optimization algorithm chooses the first counterweight's COG infinitely far away. The following values are adopted for these bounds:

$$
X_{i}^{\mathrm{m}}=-\Delta ; X_{i}^{\mathrm{M}}=\Delta ; Y_{i}^{\mathrm{m}}=-\Delta ; Y_{i}^{\mathrm{M}}=\Delta,
$$

where $\Delta>0[\mathrm{~m}]$ is a user-defined constant. It plays a critical role: in all numerical experiments with the SOCP, either $X_{1}^{*}$ or $Y_{1}^{*}$ hit the lower bound $-\Delta$ (or go to minus infinity if (10b)-(10c) are dropped). Sadler and Mayne, however, do not mention constraints like (10b)-(10c) but still obtain finite (albeit large) COG coordinates for the first counterweight. This might be attributed to the stopping criterion in the Davidon-Fletcher-Powell algorithm they use: if at some point the algorithm observes, e.g., very little improvement in the penalty function or a nearly zero penalty function gradient, it concludes that it has reached the optimum and stops, even if a slight improvement of the penalty function (by driving the first counterweight coordinates to minus infinity) is possible.

The above discussion shows that the choice of $\Delta$ is critical, but also raises the issue that Sadler and Mayne do not provide a value for it. This practical problem is solved here as follows: for each considered value of $M_{\text {shak }}^{\mathrm{M}}, \Delta$ is set equal to the maximum of $\left|X_{1}^{*}\right|,\left|Y_{1}^{*}\right|,\left|X_{3}^{*}\right|$ and $\left|Y_{3}^{*}\right|$ provided by Sadler and Mayne.

The numerical SOCP results, summarized in Table 4, illustrate that in each of the obtained SOCP optima, $\Delta$ is indeed critical: either $X_{1}^{*}$ or $Y_{1}^{*}$ hits the chosen lower bound $-\Delta$. Comparing the values of $\max \left(F_{\text {shak }}\right)$ shows that Sadler and Mayne's results are only very slightly worse than the global optimum delivered by the SOCP for $M_{\text {shak }}^{\mathrm{M}}=\{0.3509,0.2247,0.1219\}$. However, for $M_{\text {shak }}^{\mathrm{M}}=0.0853$. Sadler and Mayne's optimum is $14 \%$ worse than the SOCP optimum. This illustrates that even in a nonlinear, nonconvex program with as few as six optimization variables (Sadler and Mayne only optimized $m_{1}^{*}, X_{1}^{*}, Y_{1}^{*}, m_{3}^{*}, X_{3}^{*}$ and $X_{3}^{*}$ ), getting trapped in a local optimum is already an issue.

Besides providing better optima two additional features of the SOCP are the following. First the obtained optimum is guaranteed to be the global optimum, and hence represents a numerically proven ultimate balancing limit. This is in marked contrast with the optima of Sadler and Mayne of which inherently nothing more can be said than that they might be (or not be, one never knows) 'quite good'. Second, the results are obtained very fast: every SOCP instance is solved in five to six CPU seconds on a PentiumIV@2.8GHz processor. 


\begin{tabular}{c||c|r||r||r|r|r|r|c|c|}
\cline { 2 - 10 } & $\Delta[\mathrm{m}]$ & $M_{\text {shak }}^{\mathrm{M}}[\mathrm{N}-\mathrm{m}]$ & $\max \left(F_{\text {shak }}\right)[\mathrm{N}]$ & $m_{1}^{*}[\mathrm{~kg}]$ & $X_{1}^{*}[\mathrm{~m}]$ & $Y_{1}^{*}[\mathrm{~m}]$ & $m_{3}^{*}[\mathrm{~kg}]$ & $X_{3}^{*}[\mathrm{~m}]$ & $Y_{3}^{*}[\mathrm{~m}]$ \\
\hline \hline SaMay73 & $\ldots$ & 0.3509 & 0.00340 & 0.0010 & -135.66 & 30.5631 & 0.9990 & -0.2157 & -0.0434 \\
SOCP & 135.66 & 0.3509 & 0.00002 & 0.0010 & -135.66 & 29.3490 & 0.9990 & -0.2169 & -0.0437 \\
\hline \hline SaMay73 & $\ldots$ & 0.2247 & 0.1977 & 0.0500 & -2.8450 & -1.2312 & 0.9500 & -0.1916 & 0.0859 \\
SOCP & 2.8450 & 0.2247 & 0.1950 & 0.0449 & -2.8450 & -1.5738 & 0.9551 & -0.2024 & 0.0406 \\
\hline \hline SaMay73 & $\ldots$ & 0.1219 & 0.4038 & 0.0900 & -1.2929 & -2.7293 & 0.9100 & -0.1714 & 0.1031 \\
SOCP & 2.7293 & 0.1219 & 0.4037 & 0.0898 & -1.3684 & -2.7293 & 0.9102 & -0.1871 & 0.0747 \\
\hline \hline SaMay73 & $\ldots$ & 0.0853 & 0.5548 & 0.1100 & -1.1434 & -2.7628 & 0.8900 & -0.2174 & 0.1764 \\
SOCP & 2.7628 & 0.0853 & 0.4869 & 0.1125 & -1.5501 & -2.7628 & 0.8875 & -0.1968 & 0.0877 \\
\hline \hline
\end{tabular}

Table 4: Comparison of numerical results of Sadler and Mayne (SaMay73) and those based on the SOCP of Sec. 6.3 (SOCP). Sadler and Mayne do not specify a value for $\Delta$. For the SOCP, $\Delta$ is chosen equal to $\max \left(\left|X_{1}^{*}\right|,\left|Y_{1}^{*}\right|,\left|X_{3}^{*}\right|,\left|Y_{3}^{*}\right|\right)$, obtained by Sadler and Mayne for that particular value of $M_{s h a k}^{\mathrm{M}}$.

\section{Discussion}

It has been shown that counterweight balancing of planar crank-rocker four-bars can be cast as a convex optimization problem, that is, an SOCP. Because of (i) the associated spectacular increase in computational efficiency, and (ii) the guarantee to obtain the global optimum, it is possible to numerically prove the obtained ultimate limits of dynamic balancing in a reasonable (even very small) amount of time.

Note that there exist methods, like branch-and-bound, that guarantee to find the global optimum of any (i.e., also nonconvex) nonlinear optimization problems. The compromise, however, is efficiency. The worst-case complexity of these methods grows exponentially with the number of variables and constraints; the hope is that in practice, for the particular problem instances encountered, the method is far faster. While this favorable situation does occur, it is not typical. Even small problems, with a few tens of variables and constraints, can take a very long time (e.g., hours or days) to solve [24].

Based on a numerical example, a three-step procedure has been proposed that allows the designer to use these ultimate limits in order to make a well-balanced choice in the difficult trade-off problem that constitutes counterweight balancing. In a second example, the versatility and power of the convex optimization framework have been illustrated by reformulating the counterweight balancing method of Sadler and Mayne (1973) as a convex program and improving their numerical 
results.

However, the versatility of the proposed convex optimization framework goes beyond the two casestudies given here, which, in fact, serve as mere examples. Minimizing (or constraining to some upper limit) the (weighted sum of) maximum (absolute) or root-mean-square values of any force in a four-bar mechanism still yields a convex program. This implies that also joint forces can be considered. Generating linearly independent vector expressions for the latter is somewhat more complicated than for the shaking force, shaking moment and driving torque considered here, but still feasible using e.g. the 'boot-strap' procedure outlined in [19].

Furthermore, the applicability of the proposed framework goes beyond four-bar linkages, since it applies to any planar linkage, provided that linearly independent expressions are available for the forces and moments considered. For complicated planar mechanisms, developing such expressions by hand is cumbersome. Automated procedures, implementable in computer programs, have, however, been proposed by Elliott and Tesar [38], Kochev [39] and the first author [28].

The dynamic balancing limits obtained here are ultimate limits, in the sense that zero centroidal moment of inertia is allowed. Such point-mass counterweights are not implementable in practice. The obtained results are, however, valuable in the sense that they provide an absolute reference with which practical counterweights, such as the minimum inertia counterweights designed in Sec. 5.3, should be compared. Hence, the obtained results may play the same role as does the theoretical Carnot-efficiency when assessing the efficiency of thermodynamic processes.

The proposed procedure for converting the point-mass counterweights into practical, minimuminertia counterweights is very simple, but suboptimal. In order to obtain truly optimal, practical counterweights the 'ultimate' inertia constraint that $J_{i}^{*} \geq 0$ should be replaced by a more restrictive inertia constraint, which is to be convex in order to keep the convex structure of the overall optimization problem, and which imposes that the counterweights be implementable in practice. Some preliminary efforts along these lines can be found in [28]

\section{Acknowledgments}

Bram Demeulenaere is a Postdoctoral Fellow of the Research Foundation - Flanders (FWOVlaanderen) and has carried out part of this research as a visiting scholar (2004-2005) at the Electrical Engineering Dept. of the University of California, Los Angeles. This work has partly been carried out within the framework of project G.0462.05 'Development of a powerful dynamic optimization framework for mechanical and biomechanical systems based on convex optimization 
techniques' of the Research Foundation - Flanders (FWO - Vlaanderen) and also benefits from K.U. Leuven's Concerted Research Action GOA/99/04, and project IWT 040444 'Dynamic Balancing of Weaving Machines' of the Institute for the Promotion of Innovation by Science and Technology in Flanders (IWT). This work is also supported by K.U.Leuven-BOF EF/05/006 Center-of-Excellence Optimization in Engineering.

\section{References}

[1] R.S. Berkof and G.G. Lowen. A new method for completely force balancing simple linkages. Transactions of the ASME, Journal of Engineering for Industry, pages 21-26, 1969.

[2] G.G. Lowen, F.R. Tepper, and R.S. Berkof. Balancing of linkages-an update. Mechanism and Machine Theory, 18(3):213-220, 1983.

[3] V.H. Arakelian and M.R. Smith. Shaking force and shaking moment balancing of mechanisms: A historical review with new examples. Transactions of the ASME, Journal of Mechanical Design, 127:334-339, 2005.

[4] G.G. Lowen, F.R. Tepper, and R.S. Berkof. The quantitative influence of complete force balancing on the forces and moments of certain families of four-bar linkages. Mechanism and Machine Theory, 9:299-323, 1974.

[5] VDI2149. Blatt 1: Getriebedynamik-starrkörper mechanismen (dynamics of mechanismsrigid body mechanisms). Verein Deutscher Ingenieure - Richtlinien, 1999.

[6] R.L. Norton. Design of Machinery: An Introduction to the Synthesis and Analysis of Mechanisms and Machines (2nd Edition). McGraw-Hill, 2001.

[7] R.S. Berkof and G.G. Lowen. Theory of shaking moment optimization of force-balanced fourbar linkages. Transactions of the ASME, Journal of Engineering for Industry, pages 53-60, 1971.

[8] G.G. Lowen and R.S. Berkof. Determination of force-balanced four-bar linkages with optimum shaking moment characteristics. Transactions of the ASME, Journal of Engineering for Industry, pages 39-46, 1971.

[9] F.R. Tepper and G.G. Lowen. Shaking force optimization of four-bar linkage with adjustable constraints on ground bearing forces. Transactions of the ASME, Journal of Engineering for Industry, pages 643-651, 1975 . 
[10] J.L. Wiederrich and B. Roth. Momentum balancing of four-bar linkages. Transactions of the ASME, Journal of Engineering for Industry, pages 1289-1295, 1976.

[11] J.L. Elliott and D. Tesar. The theory of torque, shaking force and shaking moment balancing of four link mechanisms. Transactions of the ASME, Journal of Engineering for Industry, pages $715-722,1977$.

[12] R.S. Haines. Minimum rms shaking moment or driving torque of a force-balanced 4-bar linkage using feasible counterweights. Mechanism and Machine Theory, 16:185-190, 1981.

[13] B. Porter and D.J Sanger. Synthesis of dynamically optimal four-bar linkages. Proc. Conf. Mechanisms 1972, Paper No. C69/72:24-28, Institution of Mechanical Engineers, 1972.

[14] J.P. Sadler and R.W. Mayne. Balancing of mechanisms by nonlinear programming. 3rd Applied Mechanism Conference, Paper No. 29, Oklahoma State University, Stillwater, Oklahoma, 1973.

[15] M.R. Smith. Optimal balancing of planar multi-bar linkages. In Proc. 4th World Congress on the Theory of Machines and Mechanisms, pages 145-149, Newcastle-upon-Tyne, U.K., 1975.

[16] J.P. Sadler. Balancing of six-bar linkages by nonlinear programming. In Proc. 4th World Congress on the Theory of Machines and Mechanisms, pages 139-144, Newcastle-upon-Tyne, U.K., 1975 .

[17] H. Dresig and S. Schönfeld. Rechnergestützte optimierung der antriebs- und gestellkraftgrössen ebener koppelgetriebe (computer-aided optimization of driving and frame reaction forces of planar linkages). Mechanism and Machine Theory, 11(6):363-379, 1976.

[18] S.J. Tricamo and G.G. Lowen. A novel method for prescribing the maximum shaking force of a four-bar linkage with flexibility in counterweight design. Transactions of the ASME, Journal of Mechanisms, Transmissions, and Automation in Design, 105:511-519, 1983.

[19] T.W. Lee and C. Cheng. Optimum balancing of combined shaking force, shaking moment, and torque fluctuations in high-speed linkages. Transactions of the ASME, Journal of Mechanisms, Transmissions, and Automation in Design, 106:242-251, 1984.

[20] N.M. Qi and Pennestri. E. Optimum balancing of four-bar linkages-a refined algorithm. Mechanism and Machine Theory, 26(3):337-348, 1991. 
[21] Porter. B., S.S. Mohamed, and B.A. Sangolola. Genetic design of dynamically optimal fourbar linkages. In Proceedings of the 1994 ASME Mechanisms Conference, volume DE-70, pages 413-424, Minneapolis, Minnesota, 1994.

[22] G. Guo, N. Morita, and T. Torii. Optimum dynamic design of planar linkage using genetic algorithms. JSME International Journal, Series C: Mechanical Systems, Machine Elements and Manufacturing, 43(2):372-377, 2000.

[23] S. Foucault and C.M. Gosselin. Synthesis, design, and prototyping of a planar three degree-offreedom reactionless parallel mechanism. Transactions of the ASME, Journal of Mechanical Design, 126:992-999, 2004.

[24] S. Boyd and L. Vandenberghe. Convex Optimization. Cambridge University Press, 2004.

[25] F.R. Hertrich. How to balance high-speed mechanisms with minimum inertia counterweights. Machine Design, 35(6):160-164, 1963.

[26] K. Weiss and R.G. Fenton. Minimum inertia balance weight. Mech. Chem. Engng. Trans. I.E. Aust., MC8(1):93-96, 1972.

[27] J. Nocedal and S.J. Wright. Numerical Optimization. Springer-Verlag, 1999.

[28] B. Demeulenaere. Dynamic Balancing of Reciprocating Machinery With Application to Weaving Machines. PhD thesis, Dept. of Mech. Eng., K.U.Leuven, Leuven, Belgium, 2004.

[29] R.T. Rockafellar. Lagrange multipliers and duality. SIAM Review, 35:183-283, 1993.

[30] A. Ben-Tal and A. Nemirovski. Robust truss topology design via semidefinite programming. SIAM Journal on Optimization, 7(4):991-1016, 1997.

[31] A. Ben-Tal and A. Nemirovski. Lectures on Modern Convex Optimization: Analysis, Algorithms, and Engineering Applications. MPS/SIAM Series on Optimization 2. SIAM, 2001.

[32] C. Zillober. Scpip - an efficient software tool for the solution of structural optimization problems. Structural and Multidisciplinary Optimization, 24(5):362-371, 2002.

[33] M. Buss, H. Hashimoto, and J.B. Moore. Dextrous hand grasping force optimization. IEEE Transactions on Robotics and Automation, 12(3):406-418, 1996.

[34] G.N. Vanderplaats. Numerical Optimization Techniques for Engineering Design. McGrawHill, 1984. 
[35] M.S. Lobo, L. Vandenberghe, S. Boyd, and H. Lebret. Applications of second-order cone programming. Linear Algebra and its Applications, 284(1-3):193-228, 1998.

[36] C.G. Atkeson, C.H. An, and J.M. Hollerbach. Estimation of inertial parameters of manipulator loads and links. The International Journal of Robotics Research, 5(3):101-119, 1986.

[37] J. Swevers, C. Ganseman, D. Tükel, J. De Schutter, and H. Van Brussel. Optimal robot excitation and identification. IEEE Transactions on Robotics and Automation, 13:730-739, 1997.

[38] J.L. Elliott and D. Tesar. A general mass balancing method for complex planar mechanisms. Mechanism and Machine Theory, 17(2):153-172, 1982.

[39] I.S. Kochev. Contribution to the theory of torque, shaking force and shaking moment balancing of planar linkages. Mechanism and Machine Theory, 26(3):275-284, 1991.

[40] J Löfberg. Yalmip : A toolbox for modeling and optimization in matlab. In Proc. 2004 IEEE CCA/ISIC/CACSD, page Paper SaM09.1, Taipei, Taiwan, 2004.

[41] J.F. Sturm. Using sedumi 1.02, a matlab toolbox for optimization over symmetric cones. Optimization Methods and Software, 11-12:625-653, 1999. Special issue on Interior Point Methods (CD supplement with software).

[42] C.M. Gosselin, F. Vollmer, G. Côté, and Y. Wu. Synthesis and design of reactionless threedegree-of-freedom mechanisms. IEEE Transactions on Robotics and Automation, 20(2), 2004.

\section{A Linearly Independent Vector Expressions}

(11a)-(11d) are obtained based on the following expressions:

$$
\begin{aligned}
F_{\text {shak, }, x}(t) & =-\mathrm{d} L_{x} / \mathrm{d} t \\
F_{\text {shak,y }}(t) & =-\mathrm{d} L_{y} / \mathrm{d} t \\
M_{\text {shak }}(t) & =-\mathrm{d} A / \mathrm{d} t \\
M_{d r v}(t) & =\left(\mathrm{d} E_{\text {kin }} / \mathrm{d} t\right) / \dot{\phi}_{1}(t) .
\end{aligned}
$$

These expressions are valid provided that the four-bar mechanism is a kinematic chain of rigid bodies, in which no external work, gravity, friction nor springs are active. $L_{x}(t)[\mathrm{N}-\mathrm{s}]$ and $L_{y}(t)$ 
[N-s] denote the $x$ and $y$-components of the mechanism's linear momentum, whereas $A(t)[\mathrm{N}-\mathrm{m}-$ $\mathrm{s}]$ is its angular momentum some arbitrary reference point $o_{r e f} . E_{k i n}(t)[\mathrm{J}]$ is the mechanism's kinetic energy. These quantities are given by:

$$
\begin{aligned}
L_{x} & =\sum_{i=1}^{3} m_{i} \cdot \dot{x}_{i} ; \\
L_{y} & =\sum_{i=1}^{3} m_{i} \cdot \dot{y}_{i} ; \\
A & =\sum_{i=1}^{3} J_{i} \cdot \dot{\phi}_{i}+m_{i} \cdot\left(x_{i} \cdot \dot{y}_{i}-y_{i} \cdot \dot{x}_{i}\right) ; \\
E_{k i n} & =\frac{1}{2} \cdot \sum_{i=1}^{3} J_{i} \cdot \dot{\phi}_{i}^{2}+m_{i} \cdot\left(\dot{x}_{i}^{2}+\dot{y}_{i}^{2}\right) .
\end{aligned}
$$

$x_{i}[\mathrm{~m}]$ and $y_{i}[\mathrm{~m}]$ denote the $x$ and $y$-components of the position (with respect to $o_{r e f}$ ) of link $i$ 's COG,$i=1 \ldots 3$. Several methods exist to derive (11a)-(11d) from (23a)-(23d) and (22a)-(22d). Discussed here are the method of Berkof and Lowen (Sec. A.1) and the method of Elliott-TesarKochev (Sec. A.2).

\section{A.1 Berkof-Lowen Method}

This method [1] first expresses $x_{i}$ and $y_{i}$ and their derivatives as a function of the link angles $\phi_{i}$, $i=\{1,2,3\}$ and then eliminates one link angle (e.g. $\left.\phi_{2}\right)$ and its derivatives through application of the loop closure equations:

$$
\begin{aligned}
& a_{1} \cdot c_{1}+a_{2} \cdot c_{2}-a_{3} \cdot c_{3}-a_{4} \cdot c_{4}=0 \\
& a_{1} \cdot s_{1}+a_{2} \cdot s_{2}-a_{3} \cdot s_{3}-a_{4} \cdot s_{4}=0,
\end{aligned}
$$

where the shorthand notations $s_{i}=\sin \phi_{i}$ and $c_{i}=\cos \phi_{i}$ are used. This method is applied here to find the linearly independent elements of $\mathbf{e}_{f s h x}, \mathbf{e}_{f s h y}$ and $\mathbf{e}_{d r v}$, defined by (11a), (11b) and (11d):

$$
\left\{\begin{array} { l } 
{ e _ { f s h x , 1 } = c _ { 1 } \cdot \dot { \phi } _ { 1 } ^ { 2 } + s _ { 1 } \cdot \ddot { \phi } _ { 1 } ; } \\
{ e _ { f s h x , 2 } = - s _ { 1 } \cdot \dot { \phi } _ { 1 } ^ { 2 } + c _ { 1 } \cdot \ddot { \phi } _ { 1 } ; } \\
{ e _ { f s h x , 3 } = c _ { 3 } \cdot \dot { \phi } _ { 3 } ^ { 2 } + s _ { 3 } \cdot \ddot { \phi } _ { 3 } ; } \\
{ e _ { f s h x , 4 } = - s _ { 3 } \cdot \dot { \phi } _ { 3 } ^ { 2 } + c _ { 3 } \cdot \ddot { \phi } _ { 3 } ; }
\end{array} \quad \left\{\begin{array}{l}
e_{f s h y, 1}=s_{1} \cdot \dot{\phi}_{1}^{2}-c_{1} \cdot \ddot{\phi}_{1} ; \\
e_{f s h y, 2}=c_{1} \cdot \dot{\phi}_{1}^{2}+s_{1} \cdot \ddot{\phi}_{1} ; \\
e_{f s h y, 3}=s_{3} \cdot \dot{\phi}_{3}^{2}-c_{3} \cdot \ddot{\phi}_{3} ; \\
e_{f s h y, 4}=c_{3} \cdot \dot{\phi}_{3}^{2}+s_{3} \cdot \ddot{\phi}_{3} ;
\end{array}\right.\right.
$$




$$
\left\{\begin{array}{l}
e_{d r v, 1}=\ddot{\phi}_{1} ; \\
e_{d r v, 2}=\dot{\phi}_{3} \cdot \ddot{\phi}_{3} / \dot{\phi}_{1} ; \\
e_{d r v, 3}=\frac{1}{\dot{\phi}_{1}} \cdot \frac{a_{1} \cdot a_{3}}{a_{2}^{2}} \cdot\left[-s_{13} \cdot\left(\dot{\phi}_{1}-\dot{\phi}_{3}\right) \cdot \dot{\phi}_{1} \cdot \dot{\phi}_{3}+c_{13} \cdot\left(\ddot{\phi}_{1} \cdot \dot{\phi}_{3}+\dot{\phi}_{1} \cdot \ddot{\phi}_{3}\right)\right] ; \\
e_{d r v, 4}=\frac{1}{\dot{\phi}_{1}} \cdot \frac{a_{1} \cdot a_{3}}{a_{2}} \cdot\left[c_{13} \cdot\left(\dot{\phi}_{1}-\dot{\phi}_{3}\right) \cdot \dot{\phi}_{1} \cdot \dot{\phi}_{3}+s_{13} \cdot\left(\ddot{\phi}_{1} \cdot \dot{\phi}_{3}+\dot{\phi}_{1} \cdot \ddot{\phi}_{3}\right)\right] .
\end{array}\right.
$$

These elements only depend on the four-bar kinematics. If no additional linear dependencies occur (see Sec. A.3), it follows that $n_{f s h x}=n_{f s h y}=4$, while $n_{d r v}=3$ if $\dot{\phi}_{1}$ is constant, and $n_{d r v}=4$ otherwise. The elements of $\mathbf{p}_{f s h x}=\mathbf{p}_{f s h y}=\mathbf{p}_{f s h}$ and $\mathbf{p}_{d r v}$ are linear combinations (only depending on the moving link lengths) of the $\mu$-parameters:

$$
\begin{aligned}
& \left\{\begin{array}{l}
p_{f s h, 1}=\mu_{21}+a_{1} \cdot \mu_{12}-\frac{a_{1}}{a_{2}} \cdot \mu_{22} \\
p_{f s h, 2}=\mu_{31}-\frac{a_{1}}{a_{2}} \cdot \mu_{32} \\
p_{f s h, 3}=\mu_{23}+\frac{a_{3}}{a_{2}} \cdot \mu_{22} \\
p_{f s h, 4}=\mu_{33}+\frac{a_{3}}{a_{2}} \cdot \mu_{32}
\end{array}\right. \\
& \left\{\begin{array}{l}
p_{d r v, 1}=\mu_{41}-\frac{2 \cdot a_{1}^{2}}{a_{2}} \cdot \mu_{22}+\mu_{12} \cdot a_{1}^{2}+\frac{a_{1}^{2}}{a_{2}^{2}} \cdot \mu_{42} ; \\
p_{d r v, 2}=\mu_{43}+\frac{a_{3}^{2}}{a_{2}^{2}} \cdot \mu_{42} \\
p_{d r v, 3}=-\mu_{42}+a_{2} \cdot \mu_{22} \\
p_{d r v, 4}=\mu_{32}
\end{array}\right.
\end{aligned}
$$

\section{A.2 Elliott-Tesar-Kochev Method}

An alternative approach, geared towards more complicated mechanisms (for which the classical method results in cumbersome computations), was proposed by Elliott and Tesar [38] and improved by Kochev [39]. Roughly speaking, this method boils down to (i) enumerating the contributions (to the dynamic reaction considered) of each link expressed in terms of the link angles and the coordinates of the link joints (e.g the points $p, q, r$ and $s$ for the four-bar of Fig. 1), and (ii) eliminating linearly dependent terms according to a given set of rules. This method is applied here to find the linearly independent elements of $\mathbf{e}_{m s h}$, defined by (11c):

$$
\left\{\begin{array}{l}
e_{m s h, 1}=-\ddot{\phi}_{1} ; \\
e_{m s h, 2}=-\ddot{\phi}_{2} ; \\
e_{m s h, 3}=-\ddot{\phi}_{3} ; \\
e_{m s h, 4}=-x_{q} \cdot \ddot{y}_{q}+y_{q} \cdot \ddot{x}_{q} ; \\
e_{m s h, 5}=-x_{r} \cdot \ddot{y}_{r}+y_{r} \cdot \ddot{x}_{r} ; \\
e_{m s h, 6}=-\frac{1}{a_{1}} \cdot\left(x_{p} \cdot \ddot{x}_{q}-x_{q} \cdot \ddot{x}_{p}+y_{p} \cdot \ddot{y}_{q}-y_{q} \cdot \ddot{y}_{p}\right) ; \\
e_{m s h, 7}=-\frac{1}{a_{2}} \cdot\left(x_{q} \cdot \ddot{x}_{r}-x_{r} \cdot \ddot{x}_{q}+y_{q} \cdot \ddot{y}_{r}-y_{r} \cdot \ddot{y}_{q}\right) ; \\
e_{m s h, 8}=-\frac{1}{a_{3}} \cdot\left(x_{s} \cdot \ddot{x}_{r}-x_{r} \cdot \ddot{x}_{s}+y_{s} \cdot \ddot{y}_{r}-y_{r} \cdot \ddot{y}_{s}\right),
\end{array}\right.
$$


where $x_{i}[\mathrm{~m}]$ and $y_{i}[\mathrm{~m}]$ denote the $x$ and $y$-components of the position (with respect to $o_{r e f}$ ) of point $i, i=\{p, q, r, s\}$ (Fig. 1). For non-foldable mechanisms (see Sec. A.3) the eight functions $e_{m s h, k}, k=1 \ldots 8$ are linearly independent. In some special cases, however, some functions become zero or linearly dependent on other functions:

- $e_{m s h, 1}(t)=0$ if $\dot{\phi}_{1}(t)$ is constant;

- $e_{m s h, 6}(t)=0$ and $e_{m s h, 4}(t)$ is proportional to $e_{m s h, 1}(t)$ if $o_{r e f}=p$;

- $e_{m s h, 8}(t)=0$ and $e_{m s h, 5}(t)$ is proportional to $e_{m s h, 3}$ if $o_{r e f}=s$.

Hence, $n_{m s h}=5$ for the example of Sec. 5 , while $n_{m s h}=7$ for the example of Sec. 6 . The elements of $\mathbf{p}_{m s h}$ are linear combinations (only depending on the moving link lengths) of the $\mu$-parameters:

$$
\left\{\begin{array}{l}
p_{m s h, 1}=\mu_{41}-\mu_{21} \cdot a_{1} ; \\
p_{m s h, 2}=\mu_{42}-\mu_{22} \cdot a_{2} ; \\
p_{m s h, 3}=\mu_{43}-\mu_{23} \cdot a_{3} ; \\
p_{m s h, 4}=\frac{\mu_{21}}{a_{1}}+\mu_{12}-\frac{\mu_{22}}{a_{2}} ; \\
p_{m s h, 5}=\frac{\mu_{22}}{a_{2}}+\frac{\mu_{23}}{a_{3}} ; \\
p_{m s h, 6}=\frac{\mu_{31}}{a_{1}} \\
p_{m s h, 7}=\frac{\mu_{32}}{a_{2}} \\
p_{m s h, 8}=\frac{\mu_{33}}{a_{3}} .
\end{array}\right.
$$

\section{A.3 Foldable Mechanisms}

The values of $n_{d}, d=\{f s h, m s h, m d r v\}$, given in Sec. A.1 and A.2, are valid only for general four-bar mechanisms, that is, mechanisms that are not foldable. In the latter case, which implies that all links can be aligned to the base, additional linear dependencies occur as shown in [42]. For instance, if the four-bar mechanism is a parallelogram $\left(a_{1}=a_{3} ; a_{2}=a_{4}\right)$, only three (two if $\dot{\phi}_{1}$ is constant) of the eight functions $e_{m s h, k}$ are linearly independent (regardless of the choice of $\left.o_{r e f}\right)$.

\section{B Balancing Effect Index Expressions}

This appendix shows that it is possible to define auxiliary variables $\mathbf{z}_{d} \in \mathbb{R}^{n_{d}}, d=\{f s h, m s h, d r v\}$ that simultaneously satisfy

$$
\alpha_{d}=\left\|\mathbf{z}_{d}\right\|_{2} ;
$$




$$
\mathbf{z}_{d}=\mathbf{\Psi}_{d} \cdot \mathbf{p}_{d}
$$

where the matrices $\boldsymbol{\Psi}_{d} \in \mathbb{R}^{n_{d} \times n_{d}}$ only depend on the mechanism kinematics.

First consider some force $f(t)$ in the four-bar mechanism under study, and assume it is sampled at $N$ equidistant time instants over one period $T$. These samples are grouped in the force vector $\mathbf{F} \in \mathbb{R}^{N}$ :

$$
\mathbf{F}=\left[\begin{array}{llll}
f\left(T_{s}\right) & f\left(2 T_{s}\right) & \ldots & f\left(N \cdot T_{s}\right)
\end{array}\right]^{T},
$$

where $T_{s}=T / N[\mathrm{~s}]$ denotes the sample period. Using $\mathbf{F}$ and $\mathbf{F}^{\circ}$ to denote to denote the force vector after and before balancing, respectively, it follows that the balancing index is given by:

$$
\alpha=\frac{\operatorname{rms}(f)}{\operatorname{rms}\left(f^{\circ}\right)}=\frac{\|\mathbf{F}\|_{2}}{\left\|\mathbf{F}^{o}\right\|_{2}}
$$

The equality sign in this expression is only valid provided that the number of samples $N$ is high enough (say $N>500$, which is a very nonrestrictive assumption). In order to establish (25a)(25b), the LIV expression

$$
f(t)=\mathbf{e}(t)^{T} \cdot \mathbf{p}, \quad \mathbf{e}(t), \mathbf{p} \in \mathbb{R}^{n}
$$

is evaluated at each considered time instant $t_{k}=k \cdot T_{s}$ to yield

$$
\mathbf{F}=\mathbf{E} \cdot \mathbf{p}
$$

$\mathbf{E} \in \mathbb{R}^{N \times n}$ only depends on the mechanism's kinematics and is given by

$$
\mathbf{E}=\left[\begin{array}{llll}
\mathbf{e}\left(T_{s}\right) & \mathbf{e}\left(2 T_{s}\right) & \ldots & \mathbf{e}\left(N \cdot T_{s}\right)
\end{array}\right]^{T}
$$

$\mathbf{E} \in \mathbb{R}^{N \times n}$ is of full rank $n$, since the elements of $\mathbf{e}(t)$ are linearly independent. Substituting (28) into (27) subsequently yields

$$
\alpha=\frac{\|\mathbf{F}\|_{2}}{\left\|\mathbf{F}^{\mathbf{o}}\right\|_{2}}=\frac{\left(\mathbf{p}^{T} \cdot \mathbf{E}^{T} \cdot \mathbf{E} \cdot \mathbf{p}\right)^{\frac{1}{2}}}{\left\|\mathbf{F}^{\mathbf{o}}\right\|_{2}}
$$

where $\mathbf{E}^{T} \cdot \mathbf{E}$ is symmetric and positive definite. Hence, its singular value decomposition equals

$$
\mathbf{E}^{T} \cdot \mathbf{E}=\mathbf{U} \cdot \operatorname{diag}\left(\sigma_{1}, \ldots, \sigma_{n}\right) \cdot \mathbf{U}^{T}
$$

where all singular values $\sigma_{j}>0$ and $\mathbf{U} \in \mathbb{R}^{n \times n}$. Defining $\mathbf{z}$ as:

$$
\mathbf{z}=\frac{\operatorname{diag}\left(\sqrt{\sigma_{1}}, \ldots, \sqrt{\sigma_{n}}\right) \cdot \mathbf{U}^{T}}{\left\|\mathbf{F}^{\mathbf{o}}\right\|_{2}} \cdot \mathbf{p}=\mathbf{\Psi} \cdot \mathbf{p},
$$

where $\mathbf{z} \in \mathbb{R}^{n}$ and $\Psi \in \mathbb{R}^{n \times n}$, we obtain:

$$
\alpha=\|\mathbf{z}\|_{2} \text {. }
$$


Based on this result, we have:

$$
\alpha_{f s h}=\left\|\mathbf{z}_{f s h}\right\|_{2} ; \alpha_{m s h}=\left\|\mathbf{z}_{m s h}\right\|_{2} ; \alpha_{d r v}=\left\|\mathbf{z}_{d r v}\right\|_{2}
$$

where $\mathbf{z}_{d} \in \mathbb{R}^{n_{d}}, d=\{f s h, m s h, d r v\}$, and

$$
\begin{aligned}
\mathbf{z}_{f s h} & =\frac{\operatorname{diag}\left(\sqrt{\sigma_{f s h, 1}}, \ldots, \sqrt{\sigma_{f s h, n_{f s h}}}\right) \cdot \mathbf{U}_{f s h}^{T}}{\left\|\mathbf{F}_{s h a k}^{\mathrm{o}}\right\|_{2}} \cdot \mathbf{p}_{f s h}=\mathbf{\Psi}_{f s h} \cdot \mathbf{p}_{f s h} ; \\
\mathbf{z}_{m s h} & =\frac{\operatorname{diag}\left(\sqrt{\sigma_{m s h, 1}}, \ldots, \sqrt{\sigma_{m s h, n_{m s h}}}\right) \cdot \mathbf{U}_{m s h}^{T}}{\left\|\mathbf{M}_{s h a k}^{\mathrm{o}}\right\|_{2}} \cdot \mathbf{p}_{m s h}=\mathbf{\Psi}_{m s h} \cdot \mathbf{p}_{m s h} \\
\mathbf{z}_{d r v} & =\frac{\operatorname{diag}\left(\sqrt{\sigma_{d r v, 1}}, \ldots, \sqrt{\sigma_{d r v, n_{d r v}}}\right) \cdot \mathbf{U}_{d r v}^{T}}{\left\|\mathbf{M}_{d r v}^{\mathrm{o}}\right\|_{2}} \cdot \mathbf{p}_{d r v}=\boldsymbol{\Psi}_{d r v} \cdot \mathbf{p}_{d r v} .
\end{aligned}
$$

$\mathbf{U}_{d} \in \mathbb{R}^{n_{d} \times n_{d}}, d=\{f s h, m s h, d r v\}$ are determined based on:

$$
\begin{aligned}
& \mathbf{E}_{f s h x}^{T} \cdot \mathbf{E}_{f s h x}+\mathbf{E}_{f s h y}^{T} \cdot \mathbf{E}_{f s h y}=\mathbf{U}_{f s h} \cdot \operatorname{diag}\left(\sigma_{f s h, 1}, \ldots, \sigma_{f s h, n_{f s h}}\right) \cdot \mathbf{U}_{f s h}^{T} \\
& \mathbf{E}_{m s h}^{T} \cdot \mathbf{E}_{m s h}=\mathbf{U}_{m s h} \cdot \operatorname{diag}\left(\sigma_{m s h, 1}, \ldots, \sigma_{m s h, n_{m s h}}\right) \cdot \mathbf{U}_{m s h}^{T} \\
& \mathbf{E}_{d r v}^{T} \cdot \mathbf{E}_{d r v}=\mathbf{U}_{d r v} \cdot \operatorname{diag}\left(\sigma_{d r v, 1}, \ldots, \sigma_{d r v, n_{d r v}}\right) \cdot \mathbf{U}_{d r v}^{T}
\end{aligned}
$$

